\title{
The smell of lung disease: a review of the current status of electronic nose technology
}

\author{
I. G. van der Sar ${ }^{1 \dagger}$, N. Wijbenga ${ }^{1 \dagger}$, G. Nakshbandi' , J. G. J. V. Aerts ${ }^{1}$, O. C. Manintveld ${ }^{2}$, M. S. Wijsenbeek \\ M. E. Hellemons ${ }^{1+}$ and C. C. Moor ${ }^{1 *+}$ (1)
}

\begin{abstract}
There is a need for timely, accurate diagnosis, and personalised management in lung diseases. Exhaled breath reflects inflammatory and metabolic processes in the human body, especially in the lungs. The analysis of exhaled breath using electronic nose (eNose) technology has gained increasing attention in the past years. This technique has great potential to be used in clinical practice as a real-time non-invasive diagnostic tool, and for monitoring disease course and therapeutic effects. To date, multiple eNoses have been developed and evaluated in clinical studies across a wide spectrum of lung diseases, mainly for diagnostic purposes. Heterogeneity in study design, analysis techniques, and differences between eNose devices currently hamper generalization and comparison of study results. Moreover, many pilot studies have been performed, while validation and implementation studies are scarce. These studies are needed before implementation in clinical practice can be realised. This review summarises the technical aspects of available eNose devices and the available evidence for clinical application of eNose technology in different lung diseases. Furthermore, recommendations for future research to pave the way for clinical implementation of eNose technology are provided.
\end{abstract}

Keywords: Electronic nose, Breath analysis, Respiratory medicine, Personalised medicine, Machine learning, Sensor technology

\section{Background}

The field of pulmonary medicine has rapidly evolved over the last decades, with increasing knowledge about pathophysiology and aetiology leading to better targeted treatment strategies. Nevertheless, many chronic lung diseases have non-specific, often overlapping symptoms, which delays the diagnostic process and timely start of adequate treatment. Moreover, even specific disease entities can be very heterogeneous with varying phenotypes,

\footnotetext{
*Correspondence: c.moor@erasmusmc.nl

${ }^{\dagger}$ I. G. van der Sar and N. Wijbenga share first authorship

${ }^{\dagger} \mathrm{M}$. E. Hellemons and C. C. Moor share senior authorship

1 Department of Respiratory Medicine, Erasmus MC, University Medical Center, Rotterdam, The Netherlands

Full list of author information is available at the end of the article
}

and thus disease courses and optimal treatment strategies vary per patient. Accurate, non-invasive, real-time diagnostic tools and biomarkers to predict disease course and response to therapy are currently lacking in most lung diseases, but are indispensable to achieve a personalised approach for individual patients.

An emerging tool that has the potential to meet this need is an electronic nose (eNose). This device 'smells' exhaled breath for clinical diagnostics, a concept probably as old as the field of medicine itself. Exhaled breath contains thousands of molecules, also known as volatile organic compounds (VOCs). These VOCs can be divided into compounds derived from the environment (exogenous VOCs) and compounds that are the result of biological processes in the body (endogenous VOCs). original author(s) and the source, provide a link to the Creative Commons licence, and indicate if changes were made. The images or other third party material in this article are included in the article's Creative Commons licence, unless indicated otherwise in a credit line to the material. If material is not included in the article's Creative Commons licence and your intended use is not permitted by statutory regulation or exceeds the permitted use, you will need to obtain permission directly from the copyright holder. To view a copy of this licence, visit http://creativecommons.org/licenses/by/4.0/. The Creative Commons Public Domain Dedication waiver (http://creativeco mmons.org/publicdomain/zero/1.0/) applies to the data made available in this article, unless otherwise stated in a credit line to the data. 


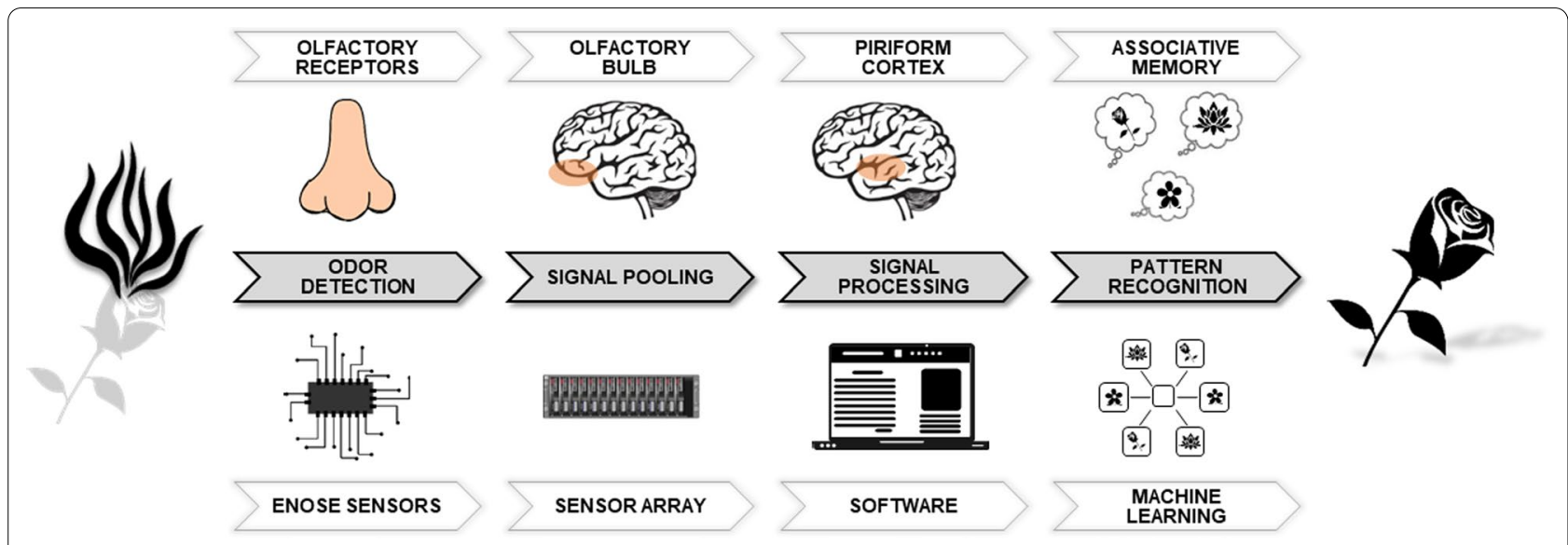

Fig. 1 Schematic comparison of eNose technology and the olfactory system [12]

Endogenous VOCs can be associated with normal physiology, but also with pathophysiological inflammatory or metabolic activity [1,2]. Identification of individual VOCs using techniques as gas chromatography or mass spectrometry is a specific but time-consuming exercise. An eNose can be used in real-time to recognise patterns of VOCs and has therefore potential as point-of-care tool in clinical practice.

The aim of this paper is to review the current clinical evidence on eNose technology in lung disease, regarding diagnosis, monitoring of disease course and therapy evaluation. In addition, technical aspects and available eNose devices are discussed.

\section{eNose technology}

In the time of Hippocrates, it was already acknowledged that exhaled breath can provide information about health conditions [3]. For instance, a sweet acetone breath odour indicates diabetes, a fishy smell suggests liver disease, and wounds with smell of grapes point towards pseudomonas infections [4]. Initial breath analysis studies were performed using gas chromatography or mass spectrometry. Throughout the last decades, more techniques were developed for breath analysis, for example ion mobility spectrometry, selected ion flow tube mass spectrometry and laser spectrometry [5]. Although these techniques became more advanced during the years and are very precise in identifying individual VOCs, they are very complex, laborious and thus not suitable as a realtime clinical practice tool.

Exhaled breath analysis by use of eNose technology is recently gaining increasing attention. An eNose is defined as "an instrument which comprises of an array of electronic-chemical sensors with partial specificity and an appropriate pattern recognition system, capable of recognising simple or complex odours" [6]. Sensors are used in eNoses to generate a singular response pattern. The sensors can generally be divided into three categories: electrical, gravimetric, and optical sensors. Each type responds to analytes (i.e. VOCs) in a specific way, and all types have a high sensitivity. Each sensor has advantages and disadvantages, without one type being superior in general. Electrical sensors consist of an electronic circuit connected to sensory materials. Upon binding with specific analytes, an electrical response is provided [7-10]. Consequently, a variation in electrical property of the sensor surface can be detected. Electrical sensors are low-cost, but are sensitive to temperature changes and have a limited sensor life [11]. Gravimetric (or mass sensitive) sensors label analytes based on changes in mass, amplitude, frequency, phase, shape, size, or position. Gravimetric sensors contain a complex circuitry and are sensitive to humidity and temperature [11]. Finally, optical sensors detect a change in colour, light intensity or emission spectra upon analyte binding. Optical sensors are insensitive to environmental changes, but are the most technically complex sensor-array systems and are not portable due to breakable optics and components. Due to the high complexity, they are more expensive than the other sensor types [11]. For each type of sensor, a more in depth explanation can be found in the Additional file 1.

Detection and recognition of odours by an eNose is similar to the functioning of the mammalian olfactory system (Fig. 1). First, an odour is detected (by olfactory receptors in a human nose or eNose sensors), which sends off various signals (to the cortex or software). Then, these signals are pooled together and processed into a pattern. This pattern can be recognised as a particular smell (e.g. a flower) [12]. As a result, an eNose can 
differentiate between diseases by analysing and comparing the smelled 'breathprints' (i.e. VOC patterns) with those previously learned. The devices are hand-held, patient friendly, easy-to-use and feasible as point-of-care test.

\section{Analysis methods}

To analyse eNose breathprints, pattern recognition by machine learning is most commonly used. A machine learning model uses algorithms which automatically improve due to experience with previously presented data. These models are in general established using a five step process: data collection, data preparation, model building, model evaluation, and model improvement. Machine learning is categorised into unsupervised, supervised, and reinforcement learning [13]. In supervised learning, the algorithms are trained with labelled data input, the desired output is thus known. On the contrary, unsupervised learning allows the algorithm to recognise patterns in the data, and groups data without providing labels. Lastly, reinforcement learning encompasses the training of the machine learning models to generate decision sequences. The latter is not used in the eNose studies reviewed in this paper.

Several machine learning models have been proposed as appropriate algorithms for modelling complex nonlinear relationships in medical research data, such as breathprints. These models include, amongst others, artificial neural networks (mimicking the structure of animal brains to model functions), ensemble neural networks (many neural networks working together to solve a problem), and support vector machines (SVM, creating a hyperplane which allows the modelling of highly complex relationships) $[14,15]$. A comparison between eNose studies show that SVM algorithm is most frequently used (10 out of 17 studies in 2019) [15]. Possibly, this is due to the fact that this is the easiest model to use for researchers new to machine learning. Another factor can be the existence of many programming languages with wellsupported libraries for SVM algorithms. SVM also possesses a high accuracy, is not very prone to overfitting, and is not overly influenced by noisy data [15]. Nonetheless, there is no consensus about the optimal model for breathprint analysis.

\section{Available eNoses}

Various eNose devices have been developed and studied in different lung diseases. Table 1 provides an overview of the specifications of devices used in studies reviewed in this paper. The choice of an eNoses device may, among others, depend on the measurement setting. For example for the BIONOTE, Cyranose 320, PEN3, and Tor Vergata eNoses the exhaled breath is captured into sample bags or cartridges which makes it possible to collect on-site and store samples for later analyses. In other settings, it could be preferable that the eNose is easily portable, like the Aeonose. The SpiroNose is the only eNose that is capable of adjusting for disturbances from ambient air using its external sensors.

The stage of development towards a clinically implemented tool differs substantially per device and disease. Before clinical implementation, each specific eNose has to be tested as a proof of concept and consecutively in substantial cohorts for each specific disease. Subsequently, data validation and clinical implementation needs to be assessed in real-life cohorts. To give more insights in the stage of development for each eNose per lung disease, we divided studies in five different stages: (1) proof of concept study; (2) cohort size of diseased participants less than fifty; (3) cohort size of diseased participants equal or more than fifty; (4) study cohort with an external validation cohort; (5) evaluation of clinical implementation. An overview of the progress per eNose and disease is visualised in Fig. 2. To the best of our knowledge, none of the devices are currently used in clinical pulmonology practice.

\section{Current clinical application}

On 21 October 2020, a systematic literature search was performed in the databases Embase, Medline (Ovid), and Cochrane Central. Search terms and selection criteria are described in the Additional file 2. Table 2 provides an overview of design and results of all studies in this review.

\section{Asthma}

Asthma is a chronic lung disease characterised by reversible airflow obstruction with airway inflammation and hyperresponsiveness. Common symptoms, such as cough, chest tightness, shortness of breath and wheezing, are variable in severity and often non-specific [17]. Various studies, both in children and adults, showed that eNose technology can differentiate asthma patients from healthy controls with a good accuracy [18-25]. Two studies also demonstrated that breathprints of asthma patients were significantly different than breathprints of chronic obstructive pulmonary disease (COPD) patients $[19,26]$. Interestingly, two studies reported better performance of eNose technology than conventional investigations (spirometry or an exhaled nitric oxide (FeNO) test) for detecting asthma. These studies were performed in patients with an established asthma diagnosis [21, 22]. Diagnostic performance further increased when eNose technology was combined with a FeNO test (accuracy 95.7\%) [21]. Moreover, even after loss of control and reaching stable disease with oral corticosteroids (OCS) treatment eNose technology could differentiate asthma 


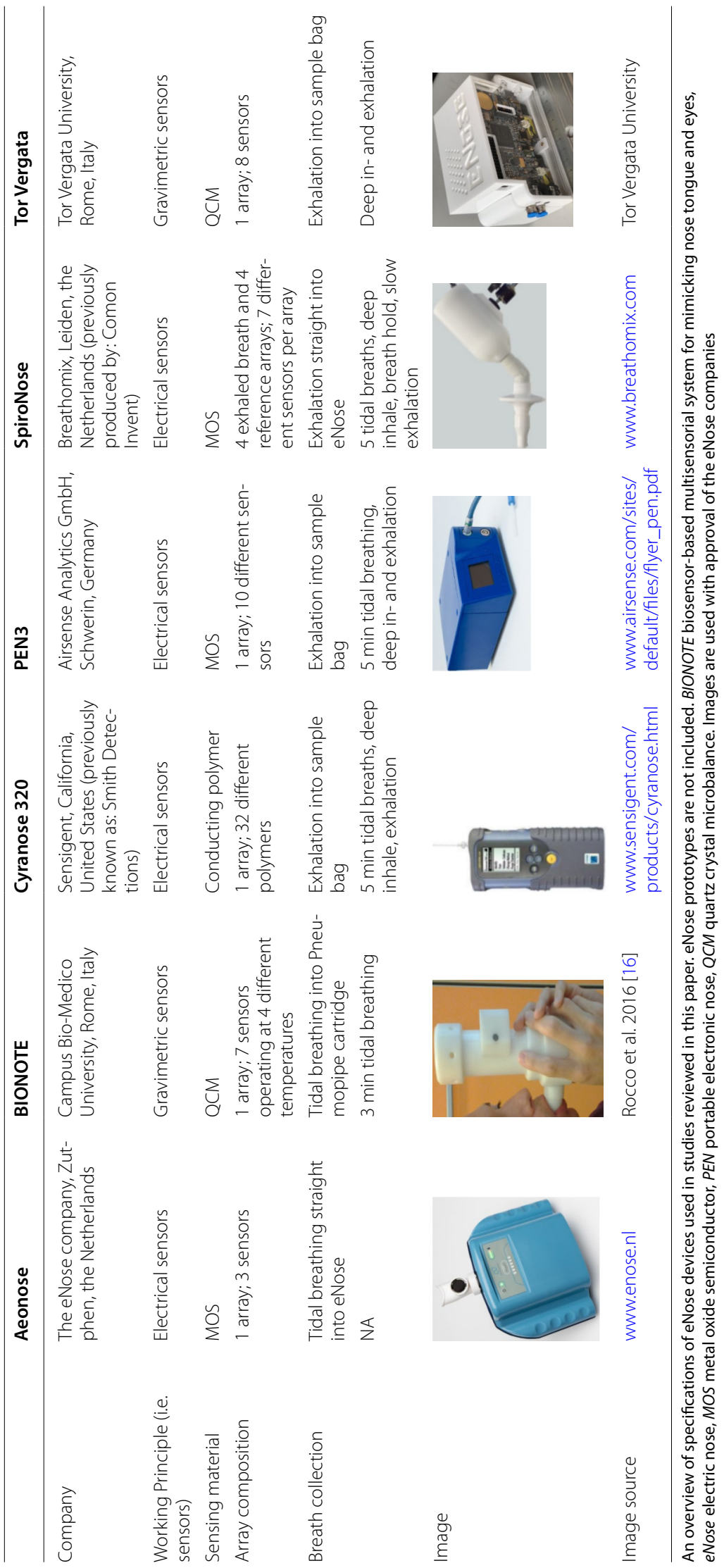




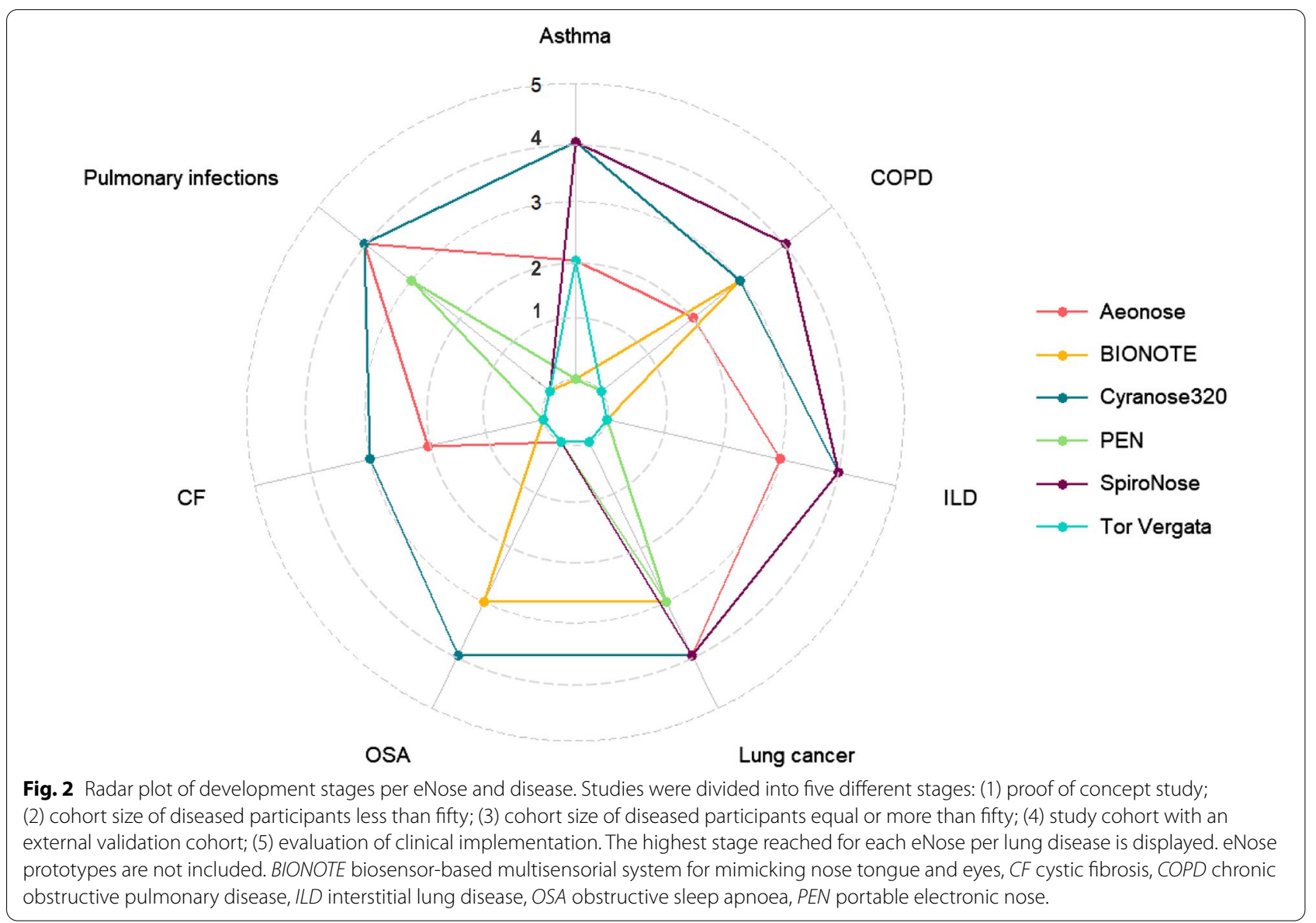

from healthy controls, while the diagnostic value of FeNO decreased. In the same study, breathprint significantly predicted response to subsequent OCS treatment, while sputum eosinophils, FeNO values and, hyperresponsiveness did not [22].

The existence of multiple asthma pheno- and endotypes with different underlying pathophysiological mechanisms is increasingly acknowledged [27]. In recent years, many eNose studies have attempted to identify different clusters of asthma patients, using both supervised and unsupervised methods [28-31]. For example, supervised clustering for eosinophilic, neutrophilic and paucigranulocytic phenotypes revealed significant differences in breathprints between groups [30]. One study identified three clusters using unsupervised breathprint analysis in a group of severe asthmatic patients, corresponding with different inflammatory profiles. During follow-up, 30 of 51 patients migrated to another cluster; migration was associated with changes in sputum eosinophil count [31]. Two other longitudinal studies showed changes in breathprint when asthma control was lost after withdrawal of corticosteroids in previously stable asthma patients, and also after recovery [22, 32]. A pilot study, in which bronchoconstriction was induced in stable asthma patients, found that changes in airway calibre did not alter breathprints. Moreover, breathprints remained stable during the day in individual patients [20]. This implies that inflammatory processes and not (acute) airway obstruction influence breathprints. Overall, these findings suggest that eNose technology is a promising tool for phenotyping and monitoring asthmatics. Longer follow-up studies are required to examine whether cluster-migration or change in breathprint are also related to actual clinical course.

A currently ongoing study is evaluating whether eNose technology can be used to predict response to monoclonal antibody therapy (NCT03988790).

\section{Paediatric asthma}

In general, the diagnosis of asthma in children is challenging. Lung function tests are often difficult to perform and do not always provide a diagnosis. Interestingly, a study in 45 children demonstrated that eNose measurements were fairly well repeatable, both in healthy and asthmatic participants [33]. 
Table 2 Literature overview eNose technology in lung disease

\begin{tabular}{|c|c|c|c|c|c|c|c|}
\hline & Study participants & $\begin{array}{l}\text { Outcome } \\
\text { measures }\end{array}$ & Results & & & eNose & $\begin{array}{l}\text { Statistical } \\
\text { breathprint } \\
\text { analysis }\end{array}$ \\
\hline \multicolumn{8}{|l|}{ Asthma } \\
\hline $\begin{array}{l}\text { Dragonieri, } \\
2007[18]\end{array}$ & $\begin{array}{l}n=20 \text { asthma } \\
\cdot n=10 \text { mild } \\
\cdot n=10 \text { severe } \\
n=20 \mathrm{HC} \\
\cdot n=10 \text { old } \\
\cdot n=10 \text { young }\end{array}$ & $\begin{array}{l}\text { Diagnostic } \\
\text { accuracy }\end{array}$ & $\begin{array}{l}\text { Mild vs young } \mathrm{HC} \\
\text { CVV } 100 \%\end{array}$ & $\begin{array}{l}\text { Severe vs old HC } \\
\text { CWV } 90 \%\end{array}$ & $\begin{array}{l}\text { Mild vs severe } \\
\text { CVV 65\% }\end{array}$ & $\begin{array}{l}\text { Cyranose } \\
320\end{array}$ & PCA; CDA \\
\hline $\begin{array}{l}\text { Fens } 2009 \\
{[19]}\end{array}$ & $\begin{array}{l}n=20 \text { asthma } \\
n=30 \text { COPD } \\
n=20 \text { non-smoking HC } \\
n=20 \text { smoking HC }\end{array}$ & $\begin{array}{l}\text { Diagnostic } \\
\text { accuracy }\end{array}$ & $\begin{array}{l}\text { COPD vs asthma } \\
\text { CVA } 96 \%\end{array}$ & $\begin{array}{l}\text { COPD vs smoking HC } \\
\text { CVA } 66 \%\end{array}$ & $\begin{array}{l}\text { Non-smoking } \\
\text { vs smoking HC } \\
\text { Not significant }\end{array}$ & $\begin{array}{l}\text { Cyranose } \\
320\end{array}$ & PCA \\
\hline $\begin{array}{l}\text { Lazar } 2010 \\
{[20]}\end{array}$ & $\begin{array}{l}n=10 \text { asthma } \\
\cdot \text { induction of bronchoconstriction } \\
\text { with methacholine or saline } \\
n=10 \text { controls }\end{array}$ & $\begin{array}{l}\text { Disease } \\
\text { course }\end{array}$ & $\begin{array}{l}\text { Bronchoconstric- } \\
\text { tion causes no } \\
\text { significant change } \\
\text { in breathprint }\end{array}$ & & & $\begin{array}{l}\text { Cyranose } \\
320\end{array}$ & $\begin{array}{l}\text { PCA; mixed } \\
\text { model } \\
\text { analysis }\end{array}$ \\
\hline $\begin{array}{l}\text { Montuschi } \\
2010[21]\end{array}$ & $\begin{array}{l}\mathrm{n}=27 \text { asthma } \\
\mathrm{n}=24 \mathrm{HC}\end{array}$ & $\begin{array}{l}\text { Diagnostic } \\
\text { accuracy }\end{array}$ & $\begin{array}{l}\text { eNose only } \\
\text { Acc } 87.5 \%\end{array}$ & $\begin{array}{l}\text { eNose+ FeNO } \\
\text { Acc } 95.8 \%\end{array}$ & & Tor Vergata & $\begin{array}{l}\text { PCA; feed- } \\
\text { forward } \\
\text { neural } \\
\text { network }\end{array}$ \\
\hline $\begin{array}{l}\text { Fens } 2011 \\
{[26]}\end{array}$ & 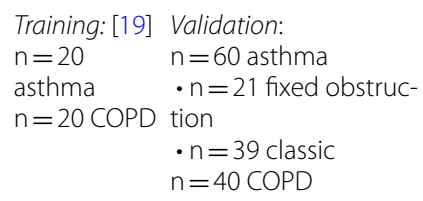 & $\begin{array}{l}\text { Diagnostic } \\
\text { accuracy }\end{array}$ & $\begin{array}{l}\text { Validation: Classic } \\
\text { asthma vs COPD } \\
\text { Sens } 85 \% \\
\text { Spec } 90 \% \\
\text { AUC } 0.93(0.84-1.00) \\
\text { Acc } 83 \%\end{array}$ & $\begin{array}{l}\text { Validation: Fixed asthma } \\
\text { vs COPD } \\
\text { Sens } 91 \% \\
\text { Spec } 90 \% \\
\text { ) AUC } 0.95(0.87-1.00) \\
\text { Acc } 88 \%\end{array}$ & $\begin{array}{l}\text { Validation: } \\
\text { Fixed vs classic } \\
\text { asthma } \\
\text { No significant } \\
\text { difference }\end{array}$ & $\begin{array}{l}\text { Cyranose } \\
320\end{array}$ & PCA; CDA \\
\hline \multirow[t]{3}{*}{$\begin{array}{l}\text { Van der } \\
\text { Schee } 2013 \\
{[22]}\end{array}$} & $\begin{array}{l}\mathrm{n}=25 \text { asthma } \\
\mathrm{n}=20 \mathrm{HC}\end{array}$ & $\begin{array}{l}\text { Diagnostic } \\
\text { accuracy }\end{array}$ & $\begin{array}{l}\text { Before OCS } \\
\text { Sens } 80.0 \% \\
\text { Spec } 65.0 \% \\
\text { AUC } 0.766 \pm 0.14\end{array}$ & $\begin{array}{l}\text { After OCS } \\
\text { Sens } 84.0 \% \\
\text { Spec } 80 \% \\
\text { AUC } 0.862 \pm 0.12\end{array}$ & $\begin{array}{l}\text { Before OCS } \\
\text { (FeNO only) } \\
\text { AUC } \\
0.738 \pm 0.15\end{array}$ & $\begin{array}{l}\text { Cyranose } \\
320\end{array}$ & PCA; CDA \\
\hline & $\begin{array}{l}\mathrm{n}=18 \text { asthma } \\
\cdot \text { maintenance ICS, stop ICS (4 weeks) } \\
\text { and OCS ( } 2 \text { weeks) }\end{array}$ & $\begin{array}{l}\text { Therapeutic } \\
\text { 5)effect }\end{array}$ & $\begin{array}{l}\text { OCS responsive } \\
\text { vs not } \\
\text { Sens } 90.9 \% \\
\text { Spec } 71.4 \% \\
\text { AUC } 0.883( \pm 0.16)\end{array}$ & & & & \\
\hline & $\begin{array}{l}\mathrm{n}=25 \text { asthma } \\
\cdot \text { maintenance ICS, stop ICS (4 weeks) } \\
\text { and OCS ( } 2 \text { weeks) } \\
\cdot \mathrm{n}=13 \text { Loss of control (LOC) }\end{array}$ & $\begin{array}{l}\text { Disease } \\
\text { s) course }\end{array}$ & $\begin{array}{l}\text { LOC vs no LOC } \\
\text { Sens } 90.9 \% \\
\text { Spec } 71.4 \% \\
\text { AUC } 0.814 \pm 0.17\end{array}$ & $\begin{array}{l}\text { Correlation sputum eos- } \\
\text { breathprint } \\
R=0.601\end{array}$ & & & \\
\hline $\begin{array}{l}\text { Plaza } 2015 \\
{[30]}\end{array}$ & $\begin{array}{l}\mathrm{n}=24 \text { eosinophilic asthma } \\
\mathrm{n}=10 \text { neutrophilic asthma } \\
\mathrm{n}=18 \text { paucigranulocytic asthma }\end{array}$ & $\begin{array}{l}\text { Diagnostic } \\
\text { accuracy }\end{array}$ & $\begin{array}{l}\text { Neutro vs pauci } \\
\text { Sens } 94 \% \\
\text { Spec } 80 \% \\
\text { AUC } 0.88 \\
\text { CVA } 89 \%\end{array}$ & $\begin{array}{l}\text { EoS vs neutro } \\
\text { Sens } 60 \% \\
\text { Spec } 79 \% \\
\text { AUC } 0.92 \\
\text { CVA } 73 \%\end{array}$ & $\begin{array}{l}\text { EoS vs pauci } \\
\text { Sens } 55 \% \\
\text { Spec } 87 \% \\
\text { AUC } 0.79 \\
\text { CVA } 74 \%\end{array}$ & $\begin{array}{l}\text { Cyranose } \\
320\end{array}$ & PCA; CDA \\
\hline $\begin{array}{l}\text { Brinkman } \\
2017[32]\end{array}$ & $\begin{array}{l}\mathrm{n}=22 \text { asthma, induced LOC } \\
\cdot \text { maintenance ICS, stop ICS ( } 8 \text { weeks) } \\
\text { and restart ICS }\end{array}$ & $\begin{array}{l}\text { Disease } \\
\text { s) course }\end{array}$ & $\begin{array}{l}\text { Baseline vs LOC } \\
\text { Acc } 95 \%\end{array}$ & $\begin{array}{l}\text { LOC vs recovery } \\
\text { Acc } 86 \%\end{array}$ & $\begin{array}{l}\text { Correlation } \\
\text { sputum eos- } \\
\text { breathprint } \\
\text { Not significant }\end{array}$ & $\begin{array}{l}\text { Cyranose } \\
320\end{array}$ & PCA \\
\hline $\begin{array}{l}\text { Bannier } \\
2019[23]\end{array}$ & $\begin{array}{l}\mathrm{n}=20 \text { asthma (age }>6 \text { years) } \\
\mathrm{n}=22 \mathrm{HC}\end{array}$ & $\begin{array}{l}\text { Diagnostic } \\
\text { accuracy }\end{array}$ & $\begin{array}{l}\text { Sens } 74 \% \\
\text { Spec } 74 \% \\
\text { AUC } 0.79\end{array}$ & & & Aeonose & ANN \\
\hline $\begin{array}{l}\text { Brinkman } \\
2019[31]\end{array}$ & $\begin{array}{l}n=78 \text { severe asthma } \\
\cdot \vec{n}=51 \text { longitudinal follow-up }\end{array}$ & Clustering & $\begin{array}{l}3 \text { clusters (baseline), } \\
\text { Differences: chronic } \\
\text { eosinophil and neutr }\end{array}$ & $\begin{array}{l}\text { acc } 93 \% \\
\text { c OCS use, percent serum } \\
\text { trophil count }\end{array}$ & $\begin{array}{l}\text { Follow-up } \\
\text { (18 months) } \\
n=21 \text { cluster } \\
\text { stable } \\
n=30 \text { migrated }\end{array}$ & $\begin{array}{l}\text { Cyranose } \\
\text { 320, Tor } \\
\text { Vergata, } \\
\text { Comon } \\
\text { dInvent }\end{array}$ & $\begin{array}{l}\text { PCA; Ward } \\
\text { clustering; } \\
\text { Non- } \\
\text { hierarchical } \\
\text { K-means } \\
\text { clustering; } \\
\text { PLS-DA; } \\
\text { PAM;Topo- } \\
\text { logical data } \\
\text { analysis }\end{array}$ \\
\hline
\end{tabular}


Table 2 (continued)

\begin{tabular}{|c|c|c|c|c|c|c|c|}
\hline & Study participants & $\begin{array}{l}\text { Outcome } \\
\text { measures }\end{array}$ & Results & & & eNose & $\begin{array}{l}\text { Statistical } \\
\text { breathprint } \\
\text { analysis }\end{array}$ \\
\hline $\begin{array}{l}\text { Cavaleiro } \\
\text { Rufo } 2019 \\
{[34]}\end{array}$ & $\begin{array}{l}n=64 \text { suspected asthma (age } \\
6-18 \text { years) } \\
\cdot n=45 \text { asthma } \\
\cdot n=29 \text { persistent } \\
\cdot n=16 \text { intermittent } \\
\cdot n=19 \text { no asthma }\end{array}$ & $\begin{array}{l}\text { Diagnostic } \\
\text { accuracy }\end{array}$ & $\begin{array}{l}\text { Asthma vs no } \\
\text { asthma } \\
\text { Sens } 77.8 \% \\
\text { Spec } 84.2 \% \\
\text { AUC } 0.81(0.69-0.93) \\
\text { Acc } 79.7 \%\end{array}$ & $\begin{array}{l}\text { Persistent vs no asthma } \\
\text { Sens } 79.7 \% \\
\text { Spec } 68.6 \% \\
\text { AUC } 0.81(0.70-0.92) \\
\text { Acc } 79.7 \%\end{array}$ & $\begin{array}{l}\text { Intermittent vs } \\
\text { no asthma } \\
\text { Not significant }\end{array}$ & $\begin{array}{l}\text { Cyranose } \\
320\end{array}$ & $\begin{array}{l}\text { PCA; } \\
\text { Hierarchical } \\
\text { clustering }\end{array}$ \\
\hline $\begin{array}{l}\text { Dragonieri } \\
2019[24]\end{array}$ & $\begin{array}{ll}\text { Training: } & \text { Validation: } \\
n=14 \text { AAR } & n=7 A A R \\
n=14 \text { rhinitis } n=7 \text { rhinitis } \\
n=14 \text { HC } \quad n=7 H C\end{array}$ & $\begin{array}{l}\text { Diagnostic } \\
\text { accuracy }\end{array}$ & $\begin{array}{l}\text { Training: } \\
\text { AAR vs HC } \\
\text { AUC } 0.87(0.70-0.97) \\
\text { CVA } 75.0 \%\end{array}$ & $\begin{array}{l}\text { Validation: } \\
\text { AAR vs HC } \\
\text { ) AUC } 0.77(0.62-0.93) \\
\text { CVA } 67.4 \%\end{array}$ & $\begin{array}{l}\text { Validation: } \\
\text { AAR vs rhinitis } \\
\text { AUC } 0.92 \\
\text { (0.84-1.00) } \\
\text { CVA } 83.1 \%\end{array}$ & $\begin{array}{l}\text { Cyranose } \\
320\end{array}$ & PCA; CDA \\
\hline $\begin{array}{l}\text { Abdel-Aziz } \\
2020[118]\end{array}$ & $\begin{array}{l}\text { Training: } \quad \text { Validation: } \\
\mathrm{n}=486 \quad \mathrm{n}=169 \text { atopic asthma } \\
\text { atopic asthma(age }>4 \text { years) } \\
\text { (age }>4 \text { years) }\end{array}$ & $\begin{array}{l}\text { Diagnostic } \\
\text { accuracy }\end{array}$ & $\begin{array}{l}\text { Training: } \\
\text { AUC 0.837-0.990 } \\
\text { Sens, spec and acc } \\
\text { only visually avail- } \\
\text { able }\end{array}$ & $\begin{array}{l}\text { Validation: } \\
\text { AUC } 0.18-0.926 \\
\text { Sens, spec and acc only } \\
\text { visually available }\end{array}$ & & $\begin{array}{l}\text { Cyranose } \\
320 \text {, Tor } \\
\text { Vergata, } \\
\text { Comon } \\
\text { Invent, } \\
\text { SpiroNose }\end{array}$ & $\begin{array}{l}\text { PLS-DA; } \\
\text { adaptive } \\
\text { least abso- } \\
\text { lute shrink- } \\
\text { age and } \\
\text { selection } \\
\text { operator; } \\
\text { gradient } \\
\text { boosting } \\
\text { machine }\end{array}$ \\
\hline $\begin{array}{l}\text { Farraia } 2020 \\
{[28]}\end{array}$ & $\begin{array}{ll}\text { Training: } & \text { Validation: } \\
\mathrm{n}=121 & \mathrm{n}=78 \text { asthma sus- } \\
\text { asthma } & \text { pected } \\
\text { suspected } & \text { (age }>6 \text { years) } \\
\text { (age }>6 \text { years) }\end{array}$ & Clustering & $\begin{array}{l}\text { Training: } 3 \text { clusters }(h \\
\text { food/drink intake } 2 ~ \\
\text { centage of asthma } \mathrm{d} \\
\text { age }<12 \text { y }\end{array}$ & $\begin{array}{l}\text { hierarchic), differences: } \\
\text { h prior to sampling, per- } \\
\text { diagnosis in group, PEF\%, }\end{array}$ & $\begin{array}{l}\text { Validation: } \\
3 \text { clusters } \\
\text { (hierarchic), dif- } \\
\text { ferences: food/ } \\
\text { drink intake } \\
2 \text { h prior to } \\
\text { sampling }\end{array}$ & $\begin{array}{l}\text { Cyranose } \\
320\end{array}$ & $\begin{array}{l}\text { Unsu- } \\
\text { pervised } \\
\text { hierarchic } \\
\text { clustering; } \\
\text { Non- } \\
\text { hierarchical } \\
\text { K-means } \\
\text { clustering; } \\
\text { PAM }\end{array}$ \\
\hline $\begin{array}{l}\text { Tenero } 2020 \\
{[25]}\end{array}$ & $\begin{array}{l}n=28 \text { asthma (age } 6-16 \text { years) } \\
\cdot n=9 \text { controlled } \\
\cdot n=7 \text { partially controlled } \\
\cdot n=12 \text { uncontrolled } \\
n=10 \mathrm{HC}\end{array}$ & $\begin{array}{l}\text { Diagnostic } \\
\text { accuracy }\end{array}$ & $\begin{array}{l}\text { HC }+ \text { controlled vs. } \\
\text { Sens } 79 \% \\
\text { Spec } 84 \% \\
\text { AUC } 0.85(0.72-0.98)\end{array}$ & partially + uncontrolled & & $\begin{array}{l}\text { Cyranose } \\
320\end{array}$ & $\begin{array}{l}\text { Penalized } \\
\text { logistic } \\
\text { regression } \\
\text { PCA }\end{array}$ \\
\hline \multicolumn{8}{|c|}{ Chronic obstructive pulmonary disease (COPD) } \\
\hline $\begin{array}{l}\text { Fens } 2011 \\
{[45]}\end{array}$ & $\begin{array}{l}\mathrm{n}=28 \text { GOLD I+ } \| \\
\cdot \text { airway inflammation (sputum } \\
\text { eosinophil cationic protein and } \\
\text { myeloperoxidase) }\end{array}$ & $\begin{array}{l}\text { Disease } \\
\text { course }\end{array}$ & $\begin{array}{l}\text { Correlation eosino- } \\
\text { phil cationic protein } \\
\text { and breathprint } \\
r=0.37\end{array}$ & $\begin{array}{l}\text { Correlation myeloperoxi- } \\
\text { dase and breathprint } \\
\text { Not significant }\end{array}$ & $\begin{array}{l}\text { Airway inflam- } \\
\text { mation vs no } \\
\text { Sens } 50-73 \% \\
\text { Spec } 77-91 \% \\
\text { AUC 0.66-0.86 }\end{array}$ & $\begin{array}{l}\text { Cyranose } \\
320\end{array}$ & PCA \\
\hline \multirow[t]{2}{*}{$\begin{array}{l}\text { Hattesohl } \\
2011[37]\end{array}$} & $\begin{array}{l}n=23 \text { COPD (pure exhaled breath, } \\
P E B) \\
n=10 \text { COPD (exhaled breath con- } \\
\text { densate, EBC) } \\
n=10 \text { HC (EBC, PEB) } \\
n=10 \text { AATd (EBC, PEB) }\end{array}$ & $\begin{array}{l}\text { Diagnostic } \\
\text { accuracy }\end{array}$ & $\begin{array}{l}\text { COPD vs HC } \\
\text { Sens } 100 \% \\
\text { Spec } 100 \% \\
\text { CV PEB } 67.6 \% \\
\text { CVV EBC } 80.5 \%\end{array}$ & $\begin{array}{l}\text { COPD vs AATd } \\
\text { Sens } 100 \% \\
\text { Spec } 100 \% \\
\text { CV PEB } 58.3 \% \\
\text { CVV EBC } 82.0 \%\end{array}$ & $\begin{array}{l}\text { HC vs AATd } \\
\text { Sens } 100 \% \\
\text { Spec } 100 \% \\
\text { CV PEB } 62.0 \% \\
\text { CV EBC } 59.5 \%\end{array}$ & $\begin{array}{l}\text { Cyranose } \\
320\end{array}$ & LDA \\
\hline & $\begin{array}{l}n=11 \text { AATd COPD (PEB) } \\
\cdot \text { augmentation therapy }\end{array}$ & $\begin{array}{l}\text { Therapeutic } \\
\text { effect }\end{array}$ & \multicolumn{3}{|l|}{$\begin{array}{l}\text { Before vs } 6 \text { d after } \\
\text { therapy } \\
\text { Sens 100\% } \\
\text { Spec 100\% } \\
\text { CWV } 53.3 \%\end{array}$} & & \\
\hline $\begin{array}{l}\text { Fens } 2013 \\
{[42]}\end{array}$ & $n=157$ COPD & Clustering & \multicolumn{3}{|c|}{$\begin{array}{l}4 \text { clusters (acc } 97.4 \%) \\
\text { Differences: airflow limitation, health related QoL, sputum pro- } \\
\text { duction, dyspnoea, smoking history, co-morbidity, radiologic } \\
\text { density, gender }\end{array}$} & $\begin{array}{l}\text { Cyranose } \\
320\end{array}$ & $\begin{array}{l}\text { Hierarchi- } \\
\text { cal cluster } \\
\text { analysis } \\
\text { Non- } \\
\text { hierarchical } \\
\text { K-means } \\
\text { clustering }\end{array}$ \\
\hline
\end{tabular}


Table 2 (continued)

\begin{tabular}{|c|c|c|c|c|c|c|c|}
\hline & Study participants & \multirow{2}{*}{$\begin{array}{l}\begin{array}{l}\text { Outcome } \\
\text { measures }\end{array} \\
\begin{array}{l}\text { Diagnostic } \\
\text { accuracy }\end{array}\end{array}$} & \multicolumn{3}{|l|}{ Results } & \multirow{2}{*}{$\begin{array}{l}\text { eNose } \\
\text { Cyranose } \\
320\end{array}$} & \multirow{2}{*}{$\begin{array}{l}\text { Statistical } \\
\text { breathprint } \\
\text { analysis }\end{array}$} \\
\hline $\begin{array}{l}\text { Sibila } 2014 \\
{[41]}\end{array}$ & $\begin{array}{l}n=10 \text { COPD bacterial colonised } \\
n=27 \text { COPD non-colonised } \\
n=13 \mathrm{HC}\end{array}$ & & $\begin{array}{l}\text { Colonised vs non- } \\
\text { colonised } \\
\text { Sens } 82 \% \\
\text { Spec } 96 \% \\
\text { AUC } 0.922 \\
\text { CVA } 89 \%\end{array}$ & $\begin{array}{l}\text { HC vs non-colonised } \\
\text { Sens } 81 \% \\
\text { Spec } 86 \% \\
\text { AUC } 0.937 \\
\text { CVA } 83 \%\end{array}$ & $\begin{array}{l}\text { HC vs colonised } \\
\text { Sens } 80 \% \\
\text { Spec } 93 \% \\
\text { AUC } 0.986 \\
\text { CVA } 87 \%\end{array}$ & & \\
\hline $\begin{array}{l}\text { Cazzola } \\
2015[38]\end{array}$ & $\begin{array}{l}n=27 \text { COPD } \\
\cdot n=8 \text { AECOPD } \geq 2 \text { per year } \\
\cdot n=19 \text { AECOPD }<2 \text { per year } \\
n=7 \text { HC }\end{array}$ & $\begin{array}{l}\text { Diagnostic } \\
\text { accuracy }\end{array}$ & $\begin{array}{l}\text { COPD vs HC } \\
\text { Sens } 96 \% \\
\text { Spec } 71 \% \\
\text { CVA } 91 \%\end{array}$ & $\begin{array}{l}\text { AECOPD } \geq 2 \text { vs }<2 \text { per } y \\
\text { Not significant }\end{array}$ & & $\begin{array}{l}\text { Prototype } \\
\text { (6 QMB sen- } \\
\text { sors) }\end{array}$ & PLS-DA \\
\hline \multicolumn{2}{|c|}{$\begin{array}{l}\text { Shafiek } 2015 n=50 \text { COPD } \\
\begin{array}{ll}\text { [39] } & \cdot n=17 \text { sputum PPM growth } \\
& n=93 \text { AECOPD } \\
& \cdot n=42 \text { sputum PPM growth } \\
n=30 \mathrm{HC}\end{array}\end{array}$} & $\begin{array}{l}\text { Diagnostic } \\
\text { accuracy }\end{array}$ & $\begin{array}{l}\text { COPD vs HC } \\
\text { Sens } 70-72 \% \\
\text { Spec } 70-73 \%\end{array}$ & \multirow{2}{*}{\multicolumn{2}{|c|}{$\begin{array}{ll}\text { COPD vs AECOPD no PPMAECOPD PPM } \\
\text { Sens } 89 \% & \text { Vs AECOPD no } \\
\text { Spec } 48 \% & \text { PPM } \\
\text { (with PPM not significant) } & \text { Sens } 88 \% \\
& \text { Spec } 60 \%\end{array}$}} & $\begin{array}{l}\text { Cyranose } \\
320\end{array}$ & LDA; SLR \\
\hline & $\begin{array}{l}n=61 \mathrm{AECOPD} \\
\cdot \text { during and } 2 \text { months after recovery }\end{array}$ & $\begin{array}{l}\text { Disease } \\
\text { y course }\end{array}$ & $\begin{array}{l}\text { During vs recovery } \\
\text { Sens } 74 \% \\
\text { Spec } 67 \%\end{array}$ & & & & \\
\hline $\begin{array}{l}\text { Van Geffen } \\
2016[46]\end{array}$ & $\begin{array}{l}n=43 \text { AECOPD } \\
\cdot n=18 \text { with viral infection } \\
\cdot n=22 \text { with bacterial infection }\end{array}$ & $\begin{array}{l}\text { Diagnostic } \\
\text { accuracy }\end{array}$ & \multicolumn{3}{|c|}{$\begin{array}{ll}\text { With vs without viral With vs without bacterial } \\
\text { infection } & \text { infection } \\
\text { Sens } 83 \% & \text { Sens } 73 \% \\
\text { Spec } 72 \% & \text { Spec } 76 \% \\
\text { AUC } 0.74 & \text { AUC } 0.72\end{array}$} & Aeonose & ANN \\
\hline $\begin{array}{l}\text { De Vries } \\
2018[43]\end{array}$ & $\begin{array}{l}\text { Validation: } \\
\mathrm{n}=114 \text { asthma/COPD }\end{array}$ & Clustering & \multicolumn{3}{|c|}{$\begin{array}{l}5 \text { clusters } \\
\text { Differences: ethnicity, systemic eosinophilia/ neutrophilia, } \\
\text { FeNO, BMI, atopy, exacerbation rate }\end{array}$} & SpiroNose & $\begin{array}{l}\text { PCA; Unsu- } \\
\text { pervised } \\
\text { Hierarchical } \\
\text { clustering }\end{array}$ \\
\hline $\begin{array}{l}\text { Finamore } \\
2018 \text { [49] }\end{array}$ & $\begin{array}{l}n=63 \text { COPD } \\
\cdot n=32 \text { n6MWD worsened } 1 \text { year } \\
\cdot n=31 \text { n6MWD stable or improved } \\
1 \text { year }\end{array}$ & $\begin{array}{l}\text { Disease } \\
\text { course }\end{array}$ & $\begin{array}{l}\text { n6MWD change } \\
\text { predicted by eNose } \\
\text { Sens } 84 \% \\
\text { Spec } 88 \% \\
\text { CVA } 86 \%\end{array}$ & \multicolumn{2}{|l|}{$\begin{array}{l}\text { n6MWD change pre- } \\
\text { dicted by eNose + GOLD } \\
\text { Sens } 81 \% \\
\text { Spec } 78 \% \\
\text { CVA } 79 \%\end{array}$} & BIONOTE & PLS-DA \\
\hline $\begin{array}{l}\text { Montuschi } \\
2018[50]\end{array}$ & $\begin{array}{l}\mathrm{n}=14 \text { COPD } \\
\cdot \text { maintenance ICS, stop ICS (4 weeks) } \\
\text { and restart ICS }\end{array}$ & $\begin{array}{l}\text { Therapeutic } \\
\text { s)effect }\end{array}$ & $\begin{array}{l}\text { Maintenance vs } \\
\text { restart ICS } \\
\text { Change in } 15 \text { of } 32 \\
\text { Cyranose sensors; } \\
3 \text { of } 8 \text { Tor Vergata } \\
\text { sensors }\end{array}$ & \multicolumn{2}{|l|}{$\begin{array}{l}\text { Maintenance vs restart } \\
\text { ICS } \\
\text { Spirometry + breathprint } \\
\text { prediction model } \\
\text { AUC } 0.857\end{array}$} & $\begin{array}{l}\text { Cyranose } \\
320, \text { Tor } \\
\text { Vergata }\end{array}$ & $\begin{array}{l}\text { Multilevel } \\
\text { PLS; KNN }\end{array}$ \\
\hline \multirow[t]{2}{*}{$\begin{array}{l}\text { Scarlata } \\
2018[44]\end{array}$} & $\begin{array}{l}\mathrm{n}=50 \text { COPD } \\
\cdot \text { standard inhalation therapy } \\
(12 \text { weeks) }\end{array}$ & $\begin{array}{l}\text { Therapeutic } \\
\text { effect }\end{array}$ & \multicolumn{3}{|l|}{$\begin{array}{l}\text { Baseline vs after } \\
12 \mathrm{w} \\
\text { Significant decline } \\
\text { in VOCs }\end{array}$} & BIONOTE & PLS-DA \\
\hline & $\mathrm{n}=50 \mathrm{COPD}$ & Clustering & \multicolumn{3}{|c|}{$\begin{array}{l}3 \text { clusters } \\
\text { Differences: BODE index, number of comorbidities, MEF75, } \\
\text { KCO, pH/pCO2 arterial blood }\end{array}$} & & $\begin{array}{l}\text { Unsu- } \\
\text { pervised } \\
\text { K-means } \\
\text { clustering }\end{array}$ \\
\hline $\begin{array}{l}\text { Van Velzen } \\
2019[47]\end{array}$ & $\begin{array}{l}n=16 \text { AECOPD } \\
\cdot \text { before, during and after recovery }\end{array}$ & $\begin{array}{l}\text { Disease } \\
\text { course }\end{array}$ & $\begin{array}{l}\text { Before vs during } \\
\text { Sens } 79 \% \\
\text { Spec } 71 \% \\
\text { CVA } 75 \%\end{array}$ & $\begin{array}{l}\text { During vs after } \\
\text { Sens } 79 \% \\
\text { Spec } 71 \% \\
\text { CVA } 75 \%\end{array}$ & $\begin{array}{l}\text { Before vs after } \\
\text { Sens } 57 \% \\
\text { Spec } 64 \% \\
\text { CVA } 61 \%\end{array}$ & $\begin{array}{l}\text { Cyranose } \\
320, \text { Tor } \\
\text { Vergata, } \\
\text { Comon } \\
\text { Invent }\end{array}$ & PCA \\
\hline $\begin{array}{l}\text { Rodríguez- } \\
\text { Aguilar } 2020 \\
\text { [40] }\end{array}$ & $\begin{array}{l}n=116 \text { COPD } \\
\cdot n=88 \text { smoking, } n=28 \text { household } \\
\text { air pollution associated } \\
\cdot n=64 \text { GOLD I-II, } n=52 \text { GOLD III-IV } \\
n=178 \mathrm{HC}\end{array}$ & $\begin{array}{l}\text { Diagnostic } \\
\text { accuracy }\end{array}$ & $\begin{array}{l}\text { COPD vs HC } \\
\text { Sens } 100 \% \\
\text { Spec } 97.8 \% \\
\text { AUC } 0.989 \\
\text { Acc } 97.8 \% \text { (CDA), } \\
100 \% \text { (SVM) }\end{array}$ & $\begin{array}{l}\text { Smoking vs air pollution } \\
\text { associated } \\
\text { Not significant }\end{array}$ & $\begin{array}{l}\text { GOLD I-II vs } \\
\text { GOLD III-IV } \\
\text { Not significant }\end{array}$ & $\begin{array}{l}\text { Cyranose } \\
320\end{array}$ & $\begin{array}{l}\text { PCA; CDA; } \\
\text { SVM }\end{array}$ \\
\hline
\end{tabular}


Table 2 (continued)

\begin{tabular}{|c|c|c|c|c|c|c|c|c|}
\hline & \multicolumn{2}{|c|}{ Study participants } & $\begin{array}{l}\text { Outcome } \\
\text { measures }\end{array}$ & \multicolumn{3}{|l|}{ Results } & eNose & $\begin{array}{l}\text { Statistical } \\
\text { breathprint } \\
\text { analysis }\end{array}$ \\
\hline \multicolumn{9}{|c|}{ Cystic fibrosis (CF) } \\
\hline $\begin{array}{l}\text { Paff } 2013 \\
{[52]}\end{array}$ & \multicolumn{2}{|c|}{$\begin{array}{l}n=25 C F \\
n=25 \text { primary ciliary dyskinesia } \\
(P C D) \\
n=23 H C\end{array}$} & $\begin{array}{l}\text { Diagnostic } \\
\text { accuracy }\end{array}$ & $\begin{array}{l}\text { CF vs HC } \\
\text { Sens } 84 \% \\
\text { Spec } 65 \% \\
\text { AUC } 0.76\end{array}$ & $\begin{array}{l}\text { CF vs PCD } \\
\text { Sens } 84 \% \\
\text { Spec } 60 \% \\
\text { AUC } 0.77\end{array}$ & \multicolumn{2}{|c|}{$\begin{array}{l}\text { Exacerbation CFCyranose } \\
\text { Sens } 89 \% \quad 320 \\
\text { Spec } 56 \% \\
\text { AUC } 0.76\end{array}$} & PCA \\
\hline $\begin{array}{l}\text { Joensen } \\
2014[53]\end{array}$ & \multicolumn{2}{|c|}{$\begin{array}{l}n=64 C F \\
\cdot n=14 \text { pseudomonas infection } \\
n=21 P C D \\
n=21 H C\end{array}$} & $\begin{array}{l}\text { Diagnostic } \\
\text { accuracy }\end{array}$ & $\begin{array}{l}\text { CF vs HC } \\
\text { Sens } 50 \% \\
\text { Spec } 95 \% \\
\text { AUC } 0.75\end{array}$ & $\begin{array}{l}\text { CF vs PCD } \\
\text { Not significant }\end{array}$ & $\begin{array}{l}\text { Pseudomonas } \\
\text { vs. non-infectec } \\
\text { CF } \\
\text { Sens } 71.4 \% \\
\text { Spec } 63.3 \% \\
\text { AUC } 0.69 \\
(0.52-0.86)\end{array}$ & $\begin{array}{l}\text { Cyranose } \\
\text { d } 320\end{array}$ & PCA \\
\hline $\begin{array}{l}\text { De Heer } \\
2016 \text { [54] }\end{array}$ & \multicolumn{2}{|c|}{$\begin{array}{l}n=9 C F \text { colonised } A \text {. fumigatus } \\
n=18 C F \text { not colonised }\end{array}$} & $\begin{array}{l}\text { Diagnostic } \\
\text { accuracy }\end{array}$ & $\begin{array}{l}\text { Sens } 78 \% \\
\text { Spec } 94 \% \\
\text { AUC } 0.80-0.89 \\
\text { CVA } 88.9 \%\end{array}$ & & & $\begin{array}{l}\text { Cyranose } \\
320\end{array}$ & PCA; CDA \\
\hline $\begin{array}{l}\text { Bannier } \\
2019[23]\end{array}$ & \multicolumn{2}{|c|}{$\begin{array}{l}\mathrm{n}=13 \mathrm{CF} \text { (age }>6 \text { years) } \\
\mathrm{n}=22 \mathrm{HC}\end{array}$} & $\begin{array}{l}\text { Diagnostic } \\
\text { accuracy }\end{array}$ & $\begin{array}{l}\text { Sens } 85 \% \\
\text { Spec } 77 \% \\
\text { AUC } 0.87\end{array}$ & & & Aeonose & ANN \\
\hline \multicolumn{9}{|c|}{ Interstitial lung disease (ILD) } \\
\hline $\begin{array}{l}\text { Dragonieri } \\
2013 \text { [58] }\end{array}$ & \multicolumn{2}{|c|}{$\begin{array}{l}n=31 \text { sarcoidosis } \\
\cdot n=11 \text { untreated } \\
\cdot n=20 \text { treated } \\
n=25 \mathrm{HC}\end{array}$} & $\begin{array}{l}\text { Diagnostic } \\
\text { accuracy }\end{array}$ & $\begin{array}{l}\text { Untreated vs HC } \\
\text { AUC 0.825 } \\
\text { CVA } 83.3 \%\end{array}$ & $\begin{array}{l}\text { Untreated vs treated } \\
\text { CVA } 74.2 \%\end{array}$ & $\begin{array}{l}\text { Treated vs HC } \\
\text { Not significant }\end{array}$ & $\begin{array}{l}\text { Cyranose } \\
320\end{array}$ & PCA; CDA \\
\hline $\begin{array}{l}\text { Yang } 2018 \\
\text { [59] }\end{array}$ & \multicolumn{2}{|c|}{$\begin{array}{ll}\text { Training: } 80 \% & \text { Valid } \\
\text { of } & n=3 \\
n=34 & \text { conic } \\
\text { pneumo- } & n=6 \\
\text { coniosis } & \\
n=64 \mathrm{HC} & \end{array}$} & $\begin{array}{l}\text { Diagnostic } \\
\text { accuracy }\end{array}$ & $\begin{array}{l}\text { Training: } \\
\text { Sens 64.3-67.9\% } \\
\text { Spec 88.0-92.0\% } \\
\text { AUC 0.89-0.91 } \\
\text { Acc } 80.8-82.1 \%\end{array}$ & $\begin{array}{l}\text { Validation: } \\
\text { Sens 33.3-66.7\% } \\
\text { Spec 71.4-78.6\% } \\
\text { AUC 0.61-0.86 } \\
\text { Acc } 65.0-70.0 \%\end{array}$ & & $\begin{array}{l}\text { Cyranose } \\
320\end{array}$ & LDA; SVM \\
\hline $\begin{array}{l}\text { Krauss } 2019 \\
{[60]}\end{array}$ & \multicolumn{2}{|c|}{$\begin{array}{l}n=174 \mathrm{ILD} \\
\cdot n=51 \mathrm{IPF} \\
\cdot n=25 \mathrm{CTD}-I L D \\
n=33 \mathrm{HC} \\
n=23 \mathrm{COPD}\end{array}$} & $\begin{array}{l}\text { Diagnostic } \\
\text { accuracy }\end{array}$ & $\begin{array}{l}\text { IPF vs HC } \\
\text { Sens } 88 \% \\
\text { Spec } 85 \% \\
\text { AUC } 0.95\end{array}$ & $\begin{array}{l}\text { CTD-ILD vs HC } \\
\text { Sens } 84 \% \\
\text { Spec } 85 \% \\
\text { AUC } 0.90\end{array}$ & $\begin{array}{l}\text { IPF vs CTD-ILD } \\
\text { Sens } 86 \% \\
\text { Spec } 64 \% \\
\text { AUC } 0.84\end{array}$ & Aeonose & ANN \\
\hline $\begin{array}{l}\text { Dragonieri } \\
2020 \text { [61] }\end{array}$ & \multicolumn{2}{|l|}{$\begin{array}{l}n=32 I P F \\
n=36 H C \\
n=33 C O P D\end{array}$} & $\begin{array}{l}\text { Diagnostic } \\
\text { accuracy }\end{array}$ & $\begin{array}{l}\text { IPF vs HC } \\
\text { AUC } 1.00(1.00-1.0 \\
\text { CVA } 98.5 \%\end{array}$ & $\begin{array}{l}\text { IPF vs COPD } \\
\text { ) AUC } 0.85(0.75-0.95) \\
\text { CVA } 80.0 \%\end{array}$ & $\begin{array}{l}\text { IPF Vs } \\
\text { COPD }+ \text { HC } \\
\text { AUC } 0.84 \\
\text { CVA } 96.1 \%\end{array}$ & $\begin{array}{l}\text { Cyranose } \\
320\end{array}$ & $\begin{array}{l}\text { PCA; CDA; } \\
\text { LDA }\end{array}$ \\
\hline $\begin{array}{l}\text { Moor } 2020 \\
{[57]}\end{array}$ & $\begin{array}{l}\text { Training: } \\
n=215 \mathrm{ILD} \\
\cdot n=57 \mathrm{IPF} \\
\cdot n=158 \\
\text { non-IPF } \\
n=32 \mathrm{HC}\end{array}$ & $\begin{array}{l}\text { Validation: } \\
n=107 \mathrm{LL} \\
\cdot n=28 \mathrm{IPF} \\
\cdot n=79 \text { non-IPF } \\
n=15 \mathrm{HC}\end{array}$ & $\begin{array}{l}\text { Diagnostic } \\
\text { accuracy }\end{array}$ & $\begin{array}{l}\text { Training + validatio } \\
\text { ILD vs HC } \\
\text { Sens } 100 \% \\
\text { Spec } 100 \% \\
\text { AUC } 1.00 \\
\text { Acc } 100 \%\end{array}$ & $\begin{array}{l}\text { Training: } \\
\text { IPF vs non-IPF ILD } \\
\text { Sens 92\% } \\
\text { Spec } 88 \% \\
\text { AUC } 0.91(0.85-0.96) \\
\text { Acc } 91 \%\end{array}$ & $\begin{array}{l}\text { Validation: } \\
\text { IPF vs non-IPF } \\
\text { ILD } \\
\text { Sens } 95 \% \\
\text { Spec } 79 \% \\
\text { AUC } 0.87 \\
(0.77-0.96) \\
\text { Acc } 91 \%\end{array}$ & SpiroNose & PLS-DA \\
\hline \multicolumn{9}{|c|}{ Lung cancer (LC) } \\
\hline $\begin{array}{l}\text { Machado } \\
2005[75]\end{array}$ & $\begin{array}{l}\text { Training: } \\
n=14 \mathrm{LC} \\
n=20 \mathrm{HC} \\
n=27 \text { other } \\
\text { lung disease }\end{array}$ & $\begin{array}{l}\text { Validation: } \\
n=14 \mathrm{LC} \\
\mathrm{n}=30 \mathrm{HC} \\
\mathrm{n}=32 \text { other lung } \\
\text { disease }\end{array}$ & $\begin{array}{l}\text { Diagnostic } \\
\text { accuracy }\end{array}$ & $\begin{array}{l}\text { Training: LC Vs } \\
\text { HC+ other } \\
\text { CVA } 71.6 \%(C D A)\end{array}$ & $\begin{array}{l}\text { Validation: LC vs } \\
\text { HC+ other } \\
\text { Sens } 71.4 \% \\
\text { Spec } 91.9 \% \\
\text { Acc } 85 \% \text { (SVM) }\end{array}$ & & $\begin{array}{l}\text { Cyranose } \\
320\end{array}$ & $\begin{array}{l}\text { SVM } \\
\text { PCA } \\
\text { CDA }\end{array}$ \\
\hline $\begin{array}{l}\text { Hubers } 201 \\
\text { [71] }\end{array}$ & $\begin{array}{l}\text { 4Training: } \\
n=20 L C \\
n=31 H C\end{array}$ & $\begin{array}{l}\text { Validation: } \\
\mathrm{n}=18 \mathrm{LC} \\
\mathrm{n}=8 \mathrm{HC}\end{array}$ & $\begin{array}{l}\text { Diagnostic } \\
\text { accuracy }\end{array}$ & $\begin{array}{l}\text { Training: } \\
\text { Sens } 80 \% \\
\text { Spec 48\% }\end{array}$ & $\begin{array}{l}\text { Validation: } \\
\text { Sens } 94 \% \\
\text { Spec } 13 \%\end{array}$ & & $\begin{array}{l}\text { Cyranose } \\
320\end{array}$ & PCA \\
\hline
\end{tabular}


Table 2 (continued)

\begin{tabular}{|c|c|c|c|c|c|c|c|c|}
\hline & \multicolumn{2}{|c|}{ Study participants } & $\begin{array}{l}\text { Outcome } \\
\text { measures }\end{array}$ & Results & & & eNose & $\begin{array}{l}\text { Statistical } \\
\text { breathprint } \\
\text { analysis }\end{array}$ \\
\hline $\begin{array}{ll}\text { Schmekel, } \\
2014 \text { [88] }\end{array}$ & \multicolumn{2}{|c|}{$\begin{array}{l}n=22 \mathrm{LC} \\
\cdot n=10 \text { survival }>1 \text { year } \\
\cdot n=12 \text { survival }<1 \text { year } \\
n=10 \mathrm{HC}\end{array}$} & $\begin{array}{l}\text { Disease } \\
\text { course }\end{array}$ & $\begin{array}{l}<1 \text { y vs } H C \\
R=0.95-0.98\end{array}$ & $\begin{array}{l}<1 \text { y vs }>1 y \\
R=0.86-0.97\end{array}$ & $\begin{array}{l}\text { Prediction } \\
\text { model survival } \\
\text { days } \\
\mathrm{R}=0.99\end{array}$ & $\begin{array}{l}\text { Applied } \\
\text { Sensor AB } \\
\text { model } 2010\end{array}$ & $\begin{array}{l}\text { PCA; PLS; } \\
\text { ANN }\end{array}$ \\
\hline $\begin{array}{l}\text { McWilliams } \\
2015 \text { [68] }\end{array}$ & \multicolumn{2}{|c|}{$\begin{array}{l}n=25 \mathrm{LC} \\
\mathrm{n}=166 \text { smoking } \mathrm{HC}\end{array}$} & $\begin{array}{l}\text { Diagnostic } \\
\text { accuracy }\end{array}$ & \multicolumn{2}{|l|}{$\begin{array}{l}\text { Sens } 84-96 \% \\
\text { Spec } 63.3-81.3 \% \\
\text { AUC } 0.84\end{array}$} & & $\begin{array}{l}\text { Cyranose } \\
320\end{array}$ & $\begin{array}{l}\text { Classifica- } \\
\text { tion and } \\
\text { regression } \\
\text { tree; DFA }\end{array}$ \\
\hline $\begin{array}{l}\text { Gasparri } \\
2016[76]\end{array}$ & $\begin{array}{l}\text { Training: } \\
n=51 \mathrm{LC} \\
n=54 \mathrm{HC}\end{array}$ & $\begin{array}{l}\text { Validation: } \\
n=21 \mathrm{LC} \\
\mathrm{n}=20 \mathrm{HC}\end{array}$ & $\begin{array}{l}\text { Diagnostic } \\
\text { accuracy }\end{array}$ & \multicolumn{2}{|c|}{$\begin{array}{ll}\text { Training + validation: Training: } \\
\text { Sens } 81 \% & \text { Sens } 90 \% \\
\text { Spec } 91 \% & \text { Spec } 100 \% \\
\text { AUC } 0.874 & \end{array}$} & $\begin{array}{l}\text { Validation: } \\
\text { Sens } 81 \% \\
\text { Spec } 100 \%\end{array}$ & $\begin{array}{l}\text { Prototype } \\
\text { (8 QMB sen- } \\
\text { sors) }\end{array}$ & PLS-DA \\
\hline $\begin{array}{l}\text { Rocco } 2016 \\
{[16]}\end{array}$ & \multicolumn{2}{|c|}{$\begin{array}{l}n=100 \text { (former) smokers } \\
\cdot n=23 \mathrm{LC}\end{array}$} & $\begin{array}{l}\text { Diagnostic } \\
\text { accuracy }\end{array}$ & $\begin{array}{l}\text { Detection LC } \\
\text { Sens } 86 \% \\
\text { Spec } 95 \% \\
\text { AUC } 0.87\end{array}$ & & & BIONOTE & $\begin{array}{l}\text { PLS-Toolbox; } \\
\text { PLS-DA }\end{array}$ \\
\hline $\begin{array}{l}\text { Van Hooren } \\
2016 \text { [81] }\end{array}$ & \multicolumn{2}{|c|}{$\begin{array}{l}n=32 L C \\
n=52 \text { head-neck SCC }\end{array}$} & $\begin{array}{l}\text { Diagnostic } \\
\text { accuracy }\end{array}$ & $\begin{array}{l}\text { Sens } 84-96 \% \\
\text { Spec } 85-88 \% \\
\text { AUC 0.88-0.98 } \\
\text { Acc } 85-93 \%\end{array}$ & & & Aeonose & ANN \\
\hline $\begin{array}{l}\text { Shlomi 2017! } \\
{[67]}\end{array}$ & \multicolumn{2}{|c|}{$\begin{array}{l}n=30 \text { benign nodule } \\
n=89 \text { LC } \\
\cdot \cdot n=16 \text { early stage } L C \\
\cdot n=53 \text { EGFR tested ( } n=19 \text { muta- } \\
\text { tion) }\end{array}$} & $\begin{array}{l}\text { Diagnostic } \\
\text { accuracy }\end{array}$ & $\begin{array}{l}\text { Early stage LC vs } \\
\text { benign } \\
\text { Sens } 75 \% \\
\text { Spec } 93.3 \% \\
\text { Acc } 87.0\end{array}$ & $\begin{array}{l}\text { EGFR mutation vs wild } \\
\text { type } \\
\text { Sens } 79.0 \% \\
\text { Spec } 85.3 \% \\
\text { Acc } 83.0 \%\end{array}$ & & $\begin{array}{l}\text { Prototype } \\
\text { ( } 40 \text { nano- } \\
\text { material- } \\
\text { sensors) }\end{array}$ & DFA \\
\hline $\begin{array}{l}\text { Tirzite } 2017 \\
{[83]}\end{array}$ & \multicolumn{2}{|c|}{$\begin{array}{l}n=165 \mathrm{LC} \\
n=79 \mathrm{HC} \\
n=91 \text { other lung disease }\end{array}$} & $\begin{array}{l}\text { Diagnostic } \\
\text { accuracy }\end{array}$ & $\begin{array}{l}\text { LC vs HC + other } \\
\text { Sens } 87.3-88.9 \% \\
\text { Spec } 66.7-71.2 \% \\
\text { CWV } 72.8 \%\end{array}$ & $\begin{array}{l}\text { LC vs HC } \\
\text { Sens } 97.8-98.8 \% \\
\text { Spec } 68.8-81.0 \% \\
\text { CWV } 69.7 \%\end{array}$ & $\begin{array}{l}\text { LC stages } \\
\text { Not significant }\end{array}$ & $\begin{array}{l}\text { Cyranose } \\
320\end{array}$ & SVM \\
\hline $\begin{array}{l}\text { Huang } 2018 \\
\text { [70] }\end{array}$ & \multicolumn{2}{|c|}{$\begin{array}{ll}\text { Training: } 80 \% & \text { Validation: } 20 \% \text { of } \\
\text { of } & n=56 \mathrm{LC} \\
\mathrm{n}=56 \mathrm{LC} & \mathrm{n}=188 \mathrm{HC} \\
\mathrm{n}=188 \mathrm{HC} & \text { External: } \\
& n=12 \mathrm{LC} \\
& n=29 \mathrm{HC}\end{array}$} & $\begin{array}{l}\text { Diagnostic } \\
\text { accuracy }\end{array}$ & $\begin{array}{l}\text { Validation: } \\
\text { LC vs HC } \\
\text { Sens } 100,92.3 \% \\
\text { Spec } 88.6,92.9 \% \\
\text { AUC } 0.96,0.95 \\
\text { Acc } 90.2,92.7 \%\end{array}$ & $\begin{array}{l}\text { External validation: } \\
\text { LC vs } \mathrm{HC} \\
\text { Sens } 75,83.3 \% \\
\text { Spec } 96.6,86.2 \% \\
\text { AUC } 0.91,0.90 \\
\text { Acc } 85.4,85.4 \%\end{array}$ & & $\begin{array}{l}\text { Cyranose } \\
320\end{array}$ & LDA; SVM \\
\hline $\begin{array}{l}\text { Van de Goor } \\
2018[73]\end{array}$ & $\begin{array}{l}\text { Training: } \\
n=52 L C \\
n=93 H C\end{array}$ & $\begin{array}{l}\text { Validation: } \\
n=8 L C n=14 H C\end{array}$ & $\begin{array}{l}\text { Diagnostic } \\
\text { accuracy }\end{array}$ & $\begin{array}{l}\text { Training: } \\
\text { Sens 83\% } \\
\text { Spec 84\% } \\
\text { AUC 0.84 } \\
\text { Acc } 83 \%\end{array}$ & $\begin{array}{l}\text { Validation: } \\
\text { Sens } 88 \% \\
\text { Spec } 86 \% \\
\text { Acc } 86 \%\end{array}$ & & Aeonose & ANN \\
\hline $\begin{array}{l}\text { Tirzite } 2019 \\
{[77]}\end{array}$ & \multicolumn{2}{|c|}{$\begin{array}{l}\mathrm{n}=119 \mathrm{LC} \text { smoker } \\
\mathrm{n}=133 \mathrm{LC} \text { non-smoker } \\
\mathrm{n}=223 \mathrm{HC}+\text { other lung disease } \\
\cdot \mathrm{n}=91 \text { smoking }\end{array}$} & $\begin{array}{l}\text { Diagnostic } \\
\text { accuracy }\end{array}$ & $\begin{array}{l}\text { LC non-smoker vs } \\
\text { HC + other } \\
\text { Sens } 96.2 \% \\
\text { Spec } 90.6 \%\end{array}$ & \multirow[t]{2}{*}{$\begin{array}{l}\text { LC smoker vs HC + other } \\
\text { Sens } 95.8 \% \\
\text { Spec } 92.3 \%\end{array}$} & & $\begin{array}{l}\text { Cyranose } \\
320\end{array}$ & LRA \\
\hline $\begin{array}{l}\text { Kononov } \\
2020[78]\end{array}$ & \multicolumn{2}{|l|}{$\begin{array}{l}n=65 \mathrm{LC} \\
\mathrm{n}=53 \mathrm{HC}\end{array}$} & $\begin{array}{l}\text { Diagnostic } \\
\text { accuracy }\end{array}$ & $\begin{array}{l}\text { Sens } 85.0-95.0 \% \\
\text { Spec } 81.2-100 \% \\
\text { CVA } 88.9-97.2 \% \\
\text { AUC } 0.95-0.98\end{array}$ & & & \multicolumn{2}{|c|}{$\begin{array}{l}\text { Prototype (6PCA; Logis- } \\
\begin{array}{ll}\text { MOS) } & \text { tic regres- } \\
& \text { sion; KNN; } \\
& \text { Random } \\
& \text { forest; LDA; } \\
& \text { SVM }\end{array}\end{array}$} \\
\hline $\begin{array}{l}\text { Krauss } 2020 \\
{[79]}\end{array}$ & \multicolumn{2}{|c|}{$\begin{array}{l}n=91 \text { LC active disease } \\
\cdot n=51 \text { incident } L C \\
n=29 L C \text { complete response } \\
n=33 \mathrm{HC} \\
n=23 \text { COPD }\end{array}$} & $\begin{array}{l}\text { Diagnostic } \\
\text { accuracy }\end{array}$ & $\begin{array}{l}\text { LC active vs HC } \\
\text { Sens } 84 \% \\
\text { Spec } 97 \% \\
\text { AUC } 0.92\end{array}$ & $\begin{array}{l}\text { Incident LC vs HC } \\
\text { Sens 88\% } \\
\text { Spec 79\% } \\
\text { AUC 89\% }\end{array}$ & & Aeonose & ANN \\
\hline \multicolumn{9}{|c|}{ Lung cancer-(non-)small cell lung cancer ((N)SCLC) } \\
\hline $\begin{array}{r}\text { Dragonieri } \\
2009[69]\end{array}$ & \multicolumn{2}{|l|}{$\begin{array}{l}n=10 \text { NSCLC } \\
n=10 \operatorname{COPD} \\
n=10 H C\end{array}$} & $\begin{array}{l}\text { Diagnostic } \\
\text { accuracy }\end{array}$ & $\begin{array}{l}\text { NSCLC vs HC } \\
\text { CWV } 90 \%\end{array}$ & $\begin{array}{l}\text { NSCLC vs COPD } \\
\text { CVV } 85 \%\end{array}$ & & $\begin{array}{l}\text { Cyranose } \\
320\end{array}$ & PCA; CDA \\
\hline
\end{tabular}


Table 2 (continued)

\begin{tabular}{|c|c|c|c|c|c|c|c|c|}
\hline & \multicolumn{2}{|c|}{ Study participants } & $\begin{array}{l}\text { Outcome } \\
\text { measures }\end{array}$ & \multicolumn{3}{|l|}{ Results } & \multirow{2}{*}{$\begin{array}{l}\text { eNose } \\
\text { Aeonose }\end{array}$} & \multirow{2}{*}{$\begin{array}{l}\begin{array}{l}\text { Statistical } \\
\text { breathprint } \\
\text { analysis }\end{array} \\
\text { ANN }\end{array}$} \\
\hline $\begin{array}{cc}\text { Kort 2018 } \\
{[72]}\end{array}$ & \multicolumn{2}{|c|}{$\begin{array}{l}\mathrm{n}=144 \mathrm{NSCLC} \\
\mathrm{n}=18 \mathrm{SCLC} \\
\mathrm{n}=85 \mathrm{HC} \\
\mathrm{n}=61 \text { suspected, } \mathrm{LC} \text { excluded }\end{array}$} & $\begin{array}{l}\text { Diagnostic } \\
\text { accuracy }\end{array}$ & $\begin{array}{l}\text { NSCLC vs HC } \\
\text { Sens } 92.2 \% \\
\text { Spec } 51.2 \% \\
\text { AUC } 0.85\end{array}$ & $\begin{array}{l}\text { NSCLC vs HC + LC } \\
\text { excluded } \\
\text { Sens } 94.4 \% \\
\text { Spec } 32.9 \% \\
\text { AUC } 0.76\end{array}$ & $\begin{array}{l}\text { SCLC vs HC } \\
\text { Sens } 90.5 \% \\
\text { Spec } 51.2 \% \\
\text { AUC } 0.86\end{array}$ & & \\
\hline $\begin{array}{l}\text { De Vries } \\
2019 \text { [87] }\end{array}$ & \multicolumn{2}{|c|}{$\begin{array}{ll}\text { Training: } & \text { Validation: } \\
\mathrm{n}=92 \mathrm{NSCLC} & \mathrm{n}=51 \mathrm{NSCLC} \\
\cdot \mathrm{n}=42 & \cdot \mathrm{n}=23 \text { response } \\
\text { response } & \cdot \mathrm{n}=28 \text { no response } \\
\cdot \boldsymbol{n}=50 \text { no } & \\
\text { response } & \end{array}$} & $\begin{array}{l}\text { Therapeutic } \\
\text { effect } \\
\text { (anti-PD-1 } \\
\text { therapy) }\end{array}$ & $\begin{array}{l}\text { Training: } \\
\text { CVV 82\% } \\
\text { AUC 0.89 (0.82-0.96) }\end{array}$ & $\begin{array}{l}\text { Validation: } \\
\text { AUC } 0.85(0.7-0.96) \\
\text { Sens } 43 \% \\
\text { Spec } 100 \%\end{array}$ & & SpiroNose & LDA \\
\hline $\begin{array}{c}\text { Mohamed } \\
2019 \text { [80] }\end{array}$ & \multicolumn{2}{|l|}{$\begin{array}{l}n=50 \mathrm{NSCLC} \\
\mathrm{n}=50 \mathrm{HC}\end{array}$} & $\begin{array}{l}\text { Diagnostic } \\
\text { accuracy }\end{array}$ & $\begin{array}{l}\text { Sens } 92.9 \% \\
\text { Spec } 90 \% \\
\text { Acc } 97.7 \%\end{array}$ & & & PEN3 & PCA; ANN \\
\hline $\begin{array}{c}\text { Kort } 2020 \\
{[74]}\end{array}$ & \multicolumn{2}{|c|}{$\begin{array}{l}n=138 \mathrm{NSCLC} \\
n=143 \text { controls } \\
\cdot n=59 \text { suspected, LC excluded } \\
\cdot n=84 \mathrm{HC}\end{array}$} & $\begin{array}{l}\text { Diagnostic } \\
\text { accuracy }\end{array}$ & $\begin{array}{l}\text { NSCLC vs controls } \\
\text { (eNose data only) } \\
\text { Sens } 94.2 \% \\
\text { Spec } 44.1 \% \\
\text { AUC } 0.75\end{array}$ & $\begin{array}{l}\text { NSCLC vs controls } \\
\text { (multivariate) } \\
\text { Sens 94.2-95.7\% } \\
\text { Spec 49.0-59.7\% } \\
\text { AUC 0.84-0.86 }\end{array}$ & & Aeonose & $\begin{array}{l}\text { ANN; } \\
\text { Multivari- } \\
\text { ate logistic } \\
\text { regression }\end{array}$ \\
\hline $\begin{array}{l}\text { Fielding } \\
2020 \text { [82] }\end{array}$ & \multicolumn{2}{|c|}{$\begin{array}{l}n=20 \text { bronchial SCC } \\
\cdot n=10 \text { in situ } \\
\cdot n=10 \text { advanced stage } \\
n=22 \text { laryngeal SCC } \\
\cdot n=12 \text { in situ } \\
\cdot n=10 \text { advanced stage } \\
n=13 \mathrm{HC}\end{array}$} & $\begin{array}{l}\text { Diagnostic } \\
\text { accuracy }\end{array}$ & $\begin{array}{l}\text { BSCC in situ vs HC } \\
\text { Sens } 77 \% \\
\text { Spec } 80 \% \\
\text { Misclassification ratel } \\
28 \%\end{array}$ & $\begin{array}{l}\text { BSCC vs LSCC adv } \\
\text { Sens } 100 \% \\
\text { Spec } 80 \% \\
\text { Misclassification rate 10\% }\end{array}$ & & $\begin{array}{l}\text { Cyranose } \\
320\end{array}$ & $\begin{array}{l}\text { Bootstrap } \\
\text { forest }\end{array}$ \\
\hline \multicolumn{9}{|c|}{ Lung cancer-Malignant Pleural Mesothelioma (MPM) } \\
\hline $\begin{array}{l}\text { Chapman } \\
2012[86]\end{array}$ & $\begin{array}{l}\text { Training: } \\
n=10 \mathrm{MPM} \\
\mathrm{n}=10 \mathrm{HC}\end{array}$ & $\begin{array}{l}\text { Validation: } \\
n=10 \text { MPM } \\
n=32 \mathrm{HC} \\
n=18 \text { benign ARD }\end{array}$ & $\begin{array}{l}\text { Diagnostic } \\
\text { accuracy }\end{array}$ & $\begin{array}{l}\text { MPM vs HC } \\
\text { Training: CVA 95\% } \\
\text { Validation: Sens 90\% } \\
\text { Spec } 91 \%\end{array}$ & $\begin{array}{l}\text { MPM vs ARD } \\
\text { Validation: Sens } 90 \% \\
\text { Spec } 83.3 \%\end{array}$ & $\begin{array}{l}\text { MPM vs ARD } \\
\text { vs HC } \\
\text { Validation: Sens } \\
90 \% \\
\text { Spec } 88 \%\end{array}$ & $\begin{array}{l}\text { Cyranose } \\
320\end{array}$ & PCA \\
\hline $\begin{array}{l}\text { Dragonieri } \\
2012 \text { [85] }\end{array}$ & \multicolumn{2}{|c|}{$\begin{array}{l}n=13 \mathrm{MPM} \\
\cdot \text { internal validation with training set: } \\
n=8 \text {, validation set: } n=5 \\
n=13 \mathrm{HC} \\
n=13 \mathrm{AEx}\end{array}$} & $\begin{array}{l}\text { Diagnostic } \\
\text { accuracy }\end{array}$ & $\begin{array}{l}\text { MPM vs HC } \\
\text { Sens } 92.3 \% \\
\text { Spec } 69.2 \% \\
\text { AUC } 0.893 \\
\text { CVA } 84.6 \% \\
\text { Validation: AUC } 0.83 \\
\text { CVA 85.0\% }\end{array}$ & $\begin{array}{l}\text { MPM vs AEx } \\
\text { Sens } 92.3 \% \\
\text { Spec } 85.7 \% \\
\text { AUC } 0.917 \\
\text { CVA } 80.8 \% \\
\text { Validation: AUC } 0.88 \\
\text { CVA } 85.9 \%\end{array}$ & $\begin{array}{l}\text { MPM vs AEx } \\
\text { vs HC } \\
\text { AUC } 0.885 \\
\text { CVA } 79.5 \%\end{array}$ & $\begin{array}{l}\text { Cyranose } \\
320\end{array}$ & PCA; CDA \\
\hline $\begin{array}{l}\text { Lamote } \\
2017 \text { [84] }\end{array}$ & \multicolumn{2}{|c|}{$\begin{array}{l}\mathrm{n}=11 \mathrm{MPM} \\
\mathrm{n}=12 \mathrm{HC} \\
\mathrm{n}=15 \text { AEx } \\
\mathrm{n}=12 \text { benign ARD }\end{array}$} & $\begin{array}{l}\text { Diagnostic } \\
\text { accuracy }\end{array}$ & $\begin{array}{l}\text { MPM vs HC } \\
\text { Sens } 66.7 \% \\
(37.7-88.4) \\
\text { Spec 63.6\% } \\
(33.7-87.2) \\
\text { AUC 0.667 (0.434- } \\
0.900) \\
\text { Acc 65.2\% } \\
(44.5-82.3)\end{array}$ & $\begin{array}{l}\text { MPM vs benign ARD } \\
\text { Sens 75.0\% (45.9-93.2) } \\
\text { Spec } 64 \%(33.7-87.2) \\
\text { AUC 0.758 (0.548-0.967) } \\
\text { Acc 48.9-85.6\% } \\
\text { (48.9-85.6) }\end{array}$ & $\begin{array}{l}\text { MPM vs benign } \\
\text { ARD + AEx } \\
\text { Sens } 81.5 \% \\
(63.7-92.9) \\
\text { Spec } 54.5 \% \\
(26.0-81.0) \\
\text { AUC } 0.747 \\
(0.582-0.913) \\
\text { Acc } 73.7 \% \\
(58.1-85.8)\end{array}$ & $\begin{array}{l}\text { Cyranose } \\
320\end{array}$ & PCA \\
\hline \multicolumn{9}{|c|}{ Pulmonary infections } \\
\hline $\begin{array}{l}\text { De Heer } \\
2016[100] \\
\end{array}$ & \multicolumn{2}{|c|}{$\begin{array}{l}n=168 \text { bottles with strain } \\
\cdot n=135 \text { bacteria }+ \text { yeast } \\
\cdot n=30 \text { medium only } \\
\cdot n=62 \text { mould (A. fumigatus and } R \text {. } \\
\text { oryzae) }\end{array}$} & $\begin{array}{l}\text { Diagnostic } \\
\text { accuracy } \\
\text { (in vitro) }\end{array}$ & $\begin{array}{l}\text { Mould vs other } \\
\text { Sens } 91.9 \% \\
\text { Spec } 95.2 \% \\
\text { AUC } 0.970(0.949- \\
0.991) \\
\text { Acc } 92.9 \%\end{array}$ & & & $\begin{array}{l}\text { Cyranose } \\
320\end{array}$ & PCA; CDA \\
\hline
\end{tabular}


Table 2 (continued)

\begin{tabular}{|c|c|c|c|c|c|c|c|c|}
\hline & \multicolumn{2}{|c|}{ Study participants } & $\begin{array}{l}\text { Outcome } \\
\text { measures }\end{array}$ & Results & & & eNose & $\begin{array}{l}\text { Statistical } \\
\text { breathprint } \\
\text { analysis }\end{array}$ \\
\hline $\begin{array}{l}\text { Suarez- } \\
\text { Cuartin } \\
2018[101]\end{array}$ & \multicolumn{2}{|c|}{$\begin{array}{l}n=73 \text { bronchiectasis } \\
\cdot n=41 \text { colonised }(n=27 \text { pseu- } \\
\text { domonas) } \\
\cdot n=32 \text { non-colonised }\end{array}$} & $\begin{array}{l}\text { Diagnostic } \\
\text { accuracy }\end{array}$ & $\begin{array}{l}\text { Colonised vs non- } \\
\text { colonised } \\
\text { AUC } 0.75 \\
\text { CVA } 72.1 \%\end{array}$ & $\begin{array}{l}\text { Pseudomonas vs other } \\
\text { PPM } \\
\text { AUC } 0.96 \\
\text { CVA } 89.2 \%\end{array}$ & $\begin{array}{l}\text { Pseudomonas } \\
\text { vs non-colo- } \\
\text { nised } \\
\text { AUC } 0.82 \\
\text { CVA } 72.7 \%\end{array}$ & $\begin{array}{l}\text { Cyranose } \\
320\end{array}$ & PCA \\
\hline \multicolumn{9}{|c|}{ Pulmonary infections - Ventilator-associated pneumonia (VAP) } \\
\hline $\begin{array}{l}\text { Hanson } \\
2005 \\
{[104]}\end{array}$ & \multicolumn{2}{|c|}{$\begin{array}{l}n=19 \text { VAP (clinical pneumonia } \\
\text { score, CPIS } \geq 6 \text { ) } \\
n=19 \text { controls }(\text { CPIS }<6)\end{array}$} & $\begin{array}{l}\text { Diagnostic } \\
\text { accuracy }\end{array}$ & $\begin{array}{l}\text { Correlation CPIS } \\
\text {-breathprint } \\
\mathrm{R}^{2}=0.81\end{array}$ & & & $\begin{array}{l}\text { Cyranose } \\
320\end{array}$ & PLS \\
\hline $\begin{array}{l}\text { Hockstein } \\
2005 \\
{[105]}\end{array}$ & \multicolumn{2}{|c|}{$\begin{array}{l}n=15 \text { VAP (pneumonia score } \geq 7) \\
n=29 \text { HC (ventilated) }\end{array}$} & $\begin{array}{l}\text { Diagnostic } \\
\text { accuracy }\end{array}$ & Acc $66-70 \%$ & & & $\begin{array}{l}\text { Cyranose } \\
320\end{array}$ & KNN \\
\hline \multicolumn{3}{|c|}{$\begin{array}{l}\text { Humphreys } n=44 \text { VAP suspected } \\
\begin{aligned} & 2011[99] \cdot 98 \text { BAL samples } \\
& \text { - Groups: gram-positive, gram-nega- } \\
& \text { tive, fungi, no growth } \\
& n=6 \text { HC (ventilated) }\end{aligned}\end{array}$} & $\begin{array}{l}\text { Diagnostic } \\
\text { accuracy } \\
\text { (in vitro) }\end{array}$ & $\begin{array}{l}\text { Differentiation } \\
\text { groups (LDA) } \\
\text { Sens } 74-95 \% \\
\text { Spec } 77-100 \% \\
\text { Acc } 83 \%\end{array}$ & $\begin{array}{l}\text { Differentiation groups } \\
\text { (cross-validation) } \\
\text { Sens } 56-84 \% \\
\text { Spec } 81-97 \% \\
\text { Acc } 70 \%\end{array}$ & & $\begin{array}{l}\text { Prototype } \\
\text { (24 MOS) }\end{array}$ & PCA; LDA \\
\hline $\begin{array}{l}\text { Schnabel } \\
2015 \\
{[106]}\end{array}$ & \multicolumn{2}{|c|}{$\begin{array}{l}n=72 \text { VAP suspected } \\
\cdot n=33 \mathrm{BAL}+ \\
\cdot n=39 \mathrm{BAL}- \\
\mathrm{n}=53 \mathrm{HC} \text { (ventilated) }\end{array}$} & $\begin{array}{l}\text { Diagnostic } \\
\text { accuracy }\end{array}$ & $\begin{array}{l}\text { BAL + VAP vs HC } \\
\text { Sens } 88 \% \\
\text { Spec 66\% } \\
\text { AUC } 0.82(0.73-0.91\end{array}$ & $\begin{array}{l}\text { BAL + Vs BAL- VAP } \\
\text { Sens } 76 \% \\
\text { Spec } 56 \% \\
\text { AUC } 0.69(0.57-0.81)\end{array}$ & & DiagNose & $\begin{array}{l}\text { Random } \\
\text { Forest; PCA }\end{array}$ \\
\hline $\begin{array}{l}\text { Chen } 2020 \\
\text { [15] }\end{array}$ & $\begin{array}{l}\text { Training: } 80 \% \\
\text { of } \\
n=33 \text { VAP } \\
n=26 \text { HC } \\
\text { (ventilated) }\end{array}$ & $\begin{array}{l}\text { Validation: } 20 \% \text { of } \\
n=33 \text { VAP } \\
n=26 \text { HC (ventilated) }\end{array}$ & $\begin{array}{l}\text { Diagnostic } \\
\text { accuracy }\end{array}$ & $\begin{array}{l}\text { Training: } \\
\text { AUC } 0.823 \\
(0.70-0.94)\end{array}$ & $\begin{array}{l}\text { Validation: } \\
\text { Sens } 79 \%( \pm 8) \\
\text { Spec } 83 \%( \pm 0) \\
\text { AUC } 0.833(0.70-0.94) \\
\text { Acc } 0.81( \pm 0.04)\end{array}$ & & $\begin{array}{l}\text { Cyranose } \\
320\end{array}$ & $\begin{array}{l}\text { KNN; Naive } \\
\text { Bayes; deci- } \\
\text { sion tree; } \\
\text { neural net- } \\
\text { work; SVM; } \\
\text { random } \\
\text { forest }\end{array}$ \\
\hline \multicolumn{9}{|c|}{ Pulmonary infections - Tuberculosis (TB) } \\
\hline $\begin{array}{l}\text { Fend } 2006 \\
\text { [109] }\end{array}$ & \multicolumn{2}{|c|}{$\begin{array}{l}n=188 T B \\
n=142 T B \text { excluded }\end{array}$} & $\begin{array}{l}\text { Diagnostic } \\
\text { accuracy } \\
\text { (in vitro) }\end{array}$ & \multicolumn{2}{|l|}{$\begin{array}{l}\text { Sens 89\% (80-97) } \\
\text { Spec } 88 \%(85-97)\end{array}$} & & $\begin{array}{l}\text { Blood- } \\
\text { hound } \\
\text { BH-114 }\end{array}$ & $\begin{array}{l}\text { PSA; DFA; } \\
\text { ANN }\end{array}$ \\
\hline $\begin{array}{l}\text { Bruins } 2013 \\
\quad[107]\end{array}$ & $\begin{array}{l}\text { Training: } \\
n=15 \text { TB } \\
n=15 \mathrm{HC}\end{array}$ & $\begin{array}{l}\text { Validation: } \\
n=34 T B \\
n=114 T B \text { excluded } \\
n=46 \mathrm{HC}\end{array}$ & $\begin{array}{l}\text { Diagnostic } \\
\text { accuracy }\end{array}$ & $\begin{array}{l}\text { Training: } \\
\text { Sens } 95.9 \% \\
(92.9-97.7) \\
\text { Spec } 98.5 \% \\
(96.2-99.4)\end{array}$ & $\begin{array}{l}\text { Validation: TB vs HC } \\
\text { Sens } 93.5 \%(91.1-95.4) \\
\text { Spec } 85.3 \%(82.7-87.5)\end{array}$ & \multicolumn{2}{|l|}{$\begin{array}{l}\text { Validation: TB vs } \\
\text { TB excl } \\
\text { Sens } 76.5 \% \\
(57.98-88.5) \\
\text { Spec } 74.8 \% \\
(64.5-82.9)\end{array}$} & ANN \\
\hline $\begin{array}{l}\text { Coronel } \\
\text { Teixeira } \\
2017 \\
{[108]}\end{array}$ & $\begin{array}{l}\text { Training: } \\
n=23 \mathrm{~TB} \\
\mathrm{n}=46 \mathrm{HC}\end{array}$ & $\begin{array}{l}\text { Validation: } \\
n=47 \mathrm{~TB} \\
\mathrm{n}=63 \\
\mathrm{HC}+\text { asthma + COPD }\end{array}$ & $\begin{array}{l}\text { Diagnostic } \\
\text { accuracy }\end{array}$ & $\begin{array}{l}\text { Training: } \\
\text { Sens 91\% } \\
\text { Spec } 93 \%\end{array}$ & $\begin{array}{l}\text { Validation: } \\
\text { Sens } 88 \% \\
\text { Spec } 92 \%\end{array}$ & & Aeonose & $\begin{array}{l}\text { Tucker } \\
\text { 3-like } \\
\text { algorithm; } \\
\text { ANN }\end{array}$ \\
\hline $\begin{array}{l}\text { Mohamed } \\
2017 \\
{[110]}\end{array}$ & \multicolumn{2}{|l|}{$\begin{array}{l}n=67 T B \\
n=56 H C\end{array}$} & $\begin{array}{l}\text { Diagnostic } \\
\text { accuracy }\end{array}$ & \multicolumn{2}{|l|}{$\begin{array}{l}\text { Sens } 98.5 \% \\
(92.1-100) \\
\text { Spec 100\% } \\
(93.5-100) \\
\text { Accuracy } 99.2 \%\end{array}$} & & PEN3 & PCA; ANN \\
\hline $\begin{array}{l}\text { Saktiawati } \\
2019 \\
{[111]}\end{array}$ & $\begin{array}{l}\text { Training: } \\
n=85 T B \\
n=97 \\
H C+T B \\
\text { excluded }\end{array}$ & $\begin{array}{l}\text { Validation: } \\
n=128 T B \\
n=159 T B \\
\text { excluded }\end{array}$ & $\begin{array}{l}\text { Diagnostic } \\
\text { accuracy }\end{array}$ & $\begin{array}{l}\text { Training: } \\
\text { Sens 85\% (75-92) } \\
\text { Spec 55\% (44-65) } \\
\text { AUC } 0.82(0.72-0.88\end{array}$ & $\begin{array}{l}\text { Validation: } \\
\text { Sens } 78 \%(70-85) \\
\text { Spec 42\% (34-50) } \\
\text { 3) AUC } 0.72(0.66-0.78)\end{array}$ & & Aeonose & ANN \\
\hline \multicolumn{3}{|c|}{$\begin{aligned} \text { Zetola } 2017 n & =51 \text { TB } \\
\text { [112] } \quad n & =20 \mathrm{HC}\end{aligned}$} & $\begin{array}{l}\text { Diagnostic } \\
\text { accuracy }\end{array}$ & $\begin{array}{l}\text { Sens } 94.1 \% \\
(83.8-98.8) \\
\text { Spec } 90.0 \% \\
(68.3-98.8)\end{array}$ & & & $\begin{array}{l}\text { Prototype } \\
\text { (QMB sen- } \\
\text { sors) }\end{array}$ & PCA; KNN \\
\hline
\end{tabular}


Table 2 (continued)

\begin{tabular}{|c|c|c|c|c|c|c|c|c|}
\hline & \multicolumn{2}{|c|}{ Study participants } & $\begin{array}{l}\text { Outcome } \\
\text { measures }\end{array}$ & \multicolumn{3}{|l|}{ Results } & eNose & $\begin{array}{l}\text { Statistical } \\
\text { breathprint } \\
\text { analysis }\end{array}$ \\
\hline \multicolumn{9}{|c|}{ Pulmonary infections-Aspergillosis } \\
\hline $\begin{array}{l}\text { De Heer } \\
2013 \\
{[102]}\end{array}$ & \multicolumn{2}{|c|}{$\begin{array}{l}n=11 \text { neutropenia } \\
\cdot n=5 \text { probable/proven aspergillosis } \\
\cdot n=6 \text { no aspergillus }\end{array}$} & $\begin{array}{l}\text { Diagnostic } \\
\text { accuracy }\end{array}$ & \multicolumn{3}{|l|}{$\begin{array}{l}\text { Sens } 100 \%(48-100) \\
\text { Spec } 83.3 \%(36-100) \\
\text { AUC } 0.933 \\
\text { CVA } 90.9 \%(59-100)\end{array}$} & $\begin{array}{l}\text { Cyranose } \\
320\end{array}$ & $P C A ; C D A$ \\
\hline $\begin{array}{l}\text { De Heer } \\
2016 \text { [54] }\end{array}$ & \multicolumn{2}{|c|}{$\begin{array}{l}n=9 C F \text { colonised } A \text {. fumigatus } \\
n=18 C F \text { not colonised }\end{array}$} & $\begin{array}{l}\text { Diagnostic } \\
\text { accuracy }\end{array}$ & \multicolumn{3}{|l|}{$\begin{array}{l}\text { Sens } 78 \% \\
\text { Spec } 94 \% \\
\text { AUC 0.80-0.89 } \\
\text { CVA } 88.9 \%\end{array}$} & $\begin{array}{l}\text { Cyranose } \\
320\end{array}$ & $P C A ; C D A$ \\
\hline \multicolumn{9}{|c|}{ Pulmonary infections - Corona Virus Disease (COVID-19) } \\
\hline $\begin{array}{l}\text { Wintjens } \\
2020 \\
{[114]}\end{array}$ & \multicolumn{2}{|c|}{$\begin{array}{l}n=219 \text { screened } \\
\cdot n=57 \text { COVID-19 positive }\end{array}$} & $\begin{array}{l}\text { Diagnostic } \\
\text { accuracy }\end{array}$ & \multicolumn{3}{|l|}{$\begin{array}{l}\text { Sens } 86 \%(74-93) \\
\text { Spec } 54 \%(46-62) \\
\text { AUC } 0.74 \\
\text { CVA } 62 \%\end{array}$} & Aeonose & ANN \\
\hline \multicolumn{9}{|c|}{ Obstructive sleep apnoea (OSA) } \\
\hline \multirow[t]{2}{*}{$\begin{array}{l}\text { Greulich } \\
2013 \text { [89] }\end{array}$} & \multicolumn{2}{|l|}{$\begin{array}{l}\mathrm{n}=40 \mathrm{OSA} \\
\mathrm{n}=20 \mathrm{HC}\end{array}$} & $\begin{array}{l}\text { Diagnostic } \\
\text { accuracy }\end{array}$ & \multicolumn{2}{|l|}{$\begin{array}{l}\text { OSA vs HC } \\
\text { Sens } 93 \% \\
\text { Spec } 70 \% \\
\text { AUC } 0.85\end{array}$} & & $\begin{array}{l}\text { Cyranose } \\
320\end{array}$ & PCA \\
\hline & \multicolumn{2}{|c|}{$\begin{array}{l}N=40 \text { OSA } \\
\cdot 3 \text { months CPAP ventilation }\end{array}$} & $\begin{array}{l}\text { Therapeutic } \\
\text { effect }\end{array}$ & \multicolumn{3}{|l|}{$\begin{array}{l}\text { Before vs after CPAP } \\
\text { Sens } 80 \% \\
\text { Spec } 65 \% \\
\text { AUC } 0.82\end{array}$} & & \\
\hline $\begin{array}{l}\text { Incalzi } 2014 \\
{[95]}\end{array}$ & \multicolumn{2}{|c|}{$\begin{array}{l}\mathrm{n}=50 \text { OSA } \\
\cdot 1 \text { night CPAP ventilation }\end{array}$} & $\begin{array}{l}\text { Therapeutic } \\
\text { effect }\end{array}$ & \multicolumn{3}{|l|}{$\begin{array}{l}\text { Change in breath- } \\
\text { print (visually dif- } \\
\text { ferent, no statistical } \\
\text { analysis) }\end{array}$} & BIONOTE & PCA; PLS-DA \\
\hline $\begin{array}{l}\text { Dragonieri } \\
2015 \text { [90] }\end{array}$ & \multicolumn{2}{|l|}{$\begin{array}{l}n=19 \text { OSA } \\
n=14 \text { obese } \\
n=20 H C\end{array}$} & $\begin{array}{l}\text { Diagnostic } \\
\text { accuracy }\end{array}$ & $\begin{array}{l}\text { Obese OSA vs HC } \\
\text { CVA\% } 97.4 \\
\text { AUC } 1.00\end{array}$ & $\begin{array}{l}\text { Obese OSA vs obese } \\
\text { CVA\% } 67.6 \\
\text { AUC } 0.77\end{array}$ & $\begin{array}{l}\text { Obese vs HC } \\
\text { CVA\% } 94.1 \\
\text { AUC } 0.94\end{array}$ & $\begin{array}{l}\text { Cyranose } \\
320\end{array}$ & $\begin{array}{l}\text { PCA; CDA; } \\
\text { KNN }\end{array}$ \\
\hline $\begin{array}{l}\text { Kunos } 2015 \\
{[96]}\end{array}$ & \multicolumn{2}{|c|}{$\begin{array}{l}n=17 \text { OSA } \\
n=9 \text { non-OSA sleep disorder } \\
n=10 \mathrm{HC} \\
\cdot 7 \mathrm{AM} \text { and } 7 \text { PM sample } \\
n=26 \mathrm{HC} \\
-7 \mathrm{AM} \text { sample }\end{array}$} & $\begin{array}{l}\text { Diagnostic } \\
\text { accuracy }\end{array}$ & $\begin{array}{l}\text { OSA 7AM vs 7PM } \\
\text { Significantly dif- } \\
\text { ferent }\end{array}$ & $\begin{array}{l}\text { Non-OSA or HC 7AM vs } \\
\text { 7PM } \\
\text { Not significantly different }\end{array}$ & $\begin{array}{l}\text { (Non-)OSA 7AM } \\
\text { vs HC 7AM } \\
\text { Significantly } \\
\text { different } \\
\text { Acc 77-81\% }\end{array}$ & $\begin{array}{l}\text { 1Cyranose } \\
320\end{array}$ & PCA \\
\hline $\begin{array}{l}\text { Dragonieri } \\
2016 \text { [92] }\end{array}$ & $\begin{array}{l}\text { Training: } \\
n=13 \text { OSA } \\
n=15 \text { COPD } \\
n=13 \\
\text { overlap }\end{array}$ & $\begin{array}{l}\text { Validation: } \\
n=6 \text { OSA } \\
n=6 \text { COPD } \\
n=6 \text { overlap }\end{array}$ & $\begin{array}{l}\text { Diagnostic } \\
\text { accuracy }\end{array}$ & $\begin{array}{l}\text { Training: } \\
\text { OSA vs overlap } \\
\text { CVA } 96.2 \% \\
\text { AUC } 0.98\end{array}$ & $\begin{array}{l}\text { Validation: } \\
\text { OSA vs overlap } \\
\text { CVA } 91.7 \% \\
\text { AUC } 1.00\end{array}$ & $\begin{array}{l}\text { Validation: } \\
\text { OSA vS COPD } \\
\text { CVA } 75 \% \\
\text { AUC } 0.83\end{array}$ & $\begin{array}{l}\text { Cyranose } \\
320\end{array}$ & PCA; CDA \\
\hline $\begin{array}{l}\text { Scarlata } \\
2017[91]\end{array}$ & $\begin{array}{l}n=40 \text { OSA } \\
\cdot n=20 \text { hypox } \\
n=20 \text { obese } \\
n=20 \text { COPD } \\
n=56 \mathrm{HC}\end{array}$ & & $\begin{array}{l}\text { Diagnostic } \\
\text { accuracy }\end{array}$ & $\begin{array}{l}\text { OSA vs HC } \\
\text { Acc } 98-100 \%\end{array}$ & $\begin{array}{l}\text { Non-hypoxic vs hypoxic } \\
\text { OSA } \\
\text { Acc } 60-80 \%\end{array}$ & $\begin{array}{l}\text { HC vs COPD } \\
\text { Acc } 100 \%\end{array}$ & BIONOTE & PLS-DA \\
\hline \multicolumn{9}{|c|}{ Other-Acute respiratory distress syndrome (ARDS) } \\
\hline $\begin{array}{l}\text { Bos } 2014 \\
{[115]}\end{array}$ & $\begin{array}{l}\text { Training: } \\
n=40 \text { ARDS } \\
n=66 \mathrm{HC}\end{array}$ & $\begin{array}{l}\text { Validation: } \\
n=18 \text { ARDS } \\
n=26 \mathrm{HC}\end{array}$ & $\begin{array}{l}\text { Diagnostic } \\
\text { accuracy }\end{array}$ & $\begin{array}{l}\text { Training: } \\
\text { Sens } 95 \% \\
\text { Spec } 42 \% \\
\text { AUC } 0.72\end{array}$ & $\begin{array}{l}\text { Validation: } \\
\text { Sens } 89 \% \\
\text { Spec } 50 \% \\
\text { AUC } 0.71\end{array}$ & & $\begin{array}{l}\text { Cyranose } \\
320\end{array}$ & $\begin{array}{l}\text { Sparse- } \\
\text { partial least } \\
\text { square } \\
\text { logistic } \\
\text { regression }\end{array}$ \\
\hline \multicolumn{9}{|c|}{ Other-Lung transplantation (LTx) } \\
\hline $\begin{array}{l}\text { Kovacs } \\
2013[117]\end{array}$ & \multicolumn{2}{|c|}{$\begin{array}{l}\mathrm{n}=16 \mathrm{LTx} \text { recipients } \\
\mathrm{n}=33 \mathrm{HC}\end{array}$} & $\begin{array}{l}\text { Diagnostic } \\
\text { accuracy }\end{array}$ & \multicolumn{2}{|l|}{$\begin{array}{l}\text { LTx recipients vs HC } \\
\text { Sens } 63 \% \\
\text { Spec } 75 \% \\
\text { AUC } 0.825\end{array}$} & & $\begin{array}{l}\text { Cyranose } \\
320\end{array}$ & $\begin{array}{l}\text { PCA; Linear } \\
\text { regression }\end{array}$ \\
\hline
\end{tabular}


Table 2 (continued)

\begin{tabular}{|c|c|c|c|c|c|c|c|}
\hline \multicolumn{2}{|c|}{ Study participants } & \multirow{2}{*}{$\begin{array}{l}\text { Outcome } \\
\text { measures }\end{array}$} & \multicolumn{3}{|l|}{ Results } & \multirow{2}{*}{$\begin{array}{l}\text { eNose } \\
\\
\text { Cyranose } \\
320\end{array}$} & \multirow{2}{*}{$\begin{array}{l}\begin{array}{l}\text { Statistical } \\
\text { breathprin } \\
\text { analysis }\end{array} \\
\begin{array}{l}\text { PCA; Linear } \\
\text { regression }\end{array}\end{array}$} \\
\hline & & & $\begin{array}{l}\text { Correlation breath- } \\
\text { print-tacrolimus } \\
\text { levels } \\
\mathrm{R}=-0.63\end{array}$ & & & & \\
\hline \multicolumn{8}{|c|}{ Other-Pulmonary embolism (PE) } \\
\hline $\begin{array}{l}\text { Fens } 2010 \\
{[116]}\end{array}$ & $\begin{array}{l}n=20 P E \\
\cdot n=7 \text { comorbidity } \\
n=20 \text { PE excluded } \\
\cdot n=13 \text { comorbidity }\end{array}$ & $\begin{array}{l}\text { Diagnostic } \\
\text { accuracy }\end{array}$ & $\begin{array}{l}\text { Comorbidity: PE vs } \\
\text { excluded } \\
\text { Acc } 65 \% \\
\text { AUC } 0.55\end{array}$ & $\begin{array}{l}\text { No comorbidity: PE vs } \\
\text { excluded } \\
\text { Acc } 85 \% \\
\text { AUC } 0.81\end{array}$ & $\begin{array}{l}\text { No comor- } \\
\text { bidity: PE } \\
\text { vs excluded } \\
\text { (breath- } \\
\text { print + Wells) } \\
\text { AUC } 0.90\end{array}$ & $\begin{array}{l}\text { Cyranose } \\
320\end{array}$ & PCA \\
\hline
\end{tabular}

An overview of eNose technology studies in lung diseases. Studies are divided per diagnosis and displayed in chronological order. Study results shown in sensitivity/ specificity, AUC and CVA (if available). In case of a training and validation set, participant numbers and results of both set are shown. All presented results are statistical significant $(p<0.05)$ unless stated otherwise

AATd alpha-1-antitrypsin deficiency, acc accuracy, $A U C$ area under the curve, $A A R$ extrinsic asthma with allergic rhinitis, $A E x$ asbestos exposure, $A N N$ artificial neural network, $A R D$ benign asbestos related disease, $B M I$ body mass index, $C D A$ canonical discriminant analysis, $C V A / C W$ cross-validated accuracy/value, $d$ days, $D F A$ discriminate function analysis, EBC exhaled breath condensate, $A E C O P D$ acute COPD exacerbation, EGFR epidermal growth factor receptor, eos eosinophils, FeNO exhaled nitric oxide test, FVC forced vital capacity, GOLD global initiative for chronic obstructive lung disease, $H C$ healthy control (not suspected for studied disease, not diagnosed with other pulmonary disease), ICS inhaled corticosteroids, IPF idiopathic pulmonary fibrosis, KNN k-nearest neighbours, LDA linear discriminant analysis, MOS metal oxide sensor, $n 6 M W D$ normalised six minute walking distance, OCS oral corticosteroids, PAM partitioning around medoids, $P C A$ principal component analysis, $P E B$ pure exhaled breath, $P L S-D A$ partial least squares discriminant analysis, $P P M$ potentially pathogenic microorganism, $Q M B$ quartz microbalance, $Q o L$ quality of life, ROC receiver operator characteristics, SCC squamous cell carcinoma ( $B$ bronchial, $L$ laryngeal), sens sensitivity, SLR Sensor Logic Relations, spec specificity, SVM support vector machines, TLC total lung capacity

Moreover, two studies showed that eNose technology distinguishes children with asthma from healthy controls $[23,25,34]$. An eNose seemed to be more accurate for diagnosing asthma than spirometry with bronchodilation only [34]. Also, uncontrolled asthma could be differentiated from controlled asthma and healthy controls [25]. Furthermore, eNose technology accurately distinguished children with persistent asthma from healthy controls, but not the ones with intermittent asthma [34]. This was possibly due to more airway inflammation reflected in the breathprints of persistent asthmatics. Hence, eNose technology could potentially facilitate easier and earlier diagnosis of asthma in children, and guide therapy in clinical practice. However, large validation studies focusing on diagnosing asthma in children are currently lacking.

\section{COPD}

Although COPD is one of the major causes of death worldwide, epidemiological studies indicate that it remains largely underdiagnosed [35]. COPD is a complex, heterogeneous disease with several phenotypes, which can overlap with asthma and pulmonary infections, among others. Furthermore, the diagnosis is delayed in patients whose symptoms are attributed to (undiagnosed) heart failure [36]. Hence, there is an unmet clinical need for accurate timely diagnosis. Also better disease course prediction and therapy guidance is warranted.
Several studies have evaluated the ability of eNose technology to diagnose COPD. Exhaled breath analysis discriminated between COPD and (smoking) healthy controls with an accuracy of 66-100\% [19, 37-41]. Even though these are promising results, most studies were relatively small and lacked a validation cohort. Several studies aimed to distinguish subgroups within COPD by performing unsupervised analyses on breathprint data [42-44]. De Vries et al. performed unsupervised cluster analysis in a combined group of asthma and COPD patients [43]. Interestingly, they identified and validated five clusters which mainly differed based on clinical and inflammatory characteristics (eosinophil and neutrophil count) rather than diagnosis. Two other studies identified 3-4 unsupervised clusters based on breathprint data. The clusters differed regarding several clinical and demographic features [42, 44]. However, in both studies, clusters were determined by different clinical parameters, showing the need for further (validation) studies. A recent study indicated that breathprints of patients with COPD associated with air pollution did not differ from smoking-associated COPD [40]. Also, no differences in breathprint between Global Initiative for Chronic Obstructive Lung Disease (GOLD) stage I-II versus GOLD stage III-IV were detected in another study [40]. The breathprint of patients with smoking-related COPD and patients with alpha-1-antitripsin, however, could be distinguished with an accuracy of $82 \%$ in a small singlecentre study [37]. 
eNose technology can theoretically be useful in early detection of inflammation and acute exacerbation of COPD (AECOPD), as inflammatory processes influence breathprints. This hypothesis was confirmed in a crosssectional study evaluating the association of breathprints with different inflammation markers in sputum; eNose breathprints highly correlated with inflammatory activity [45]. In patients with an AECOPD, presence of viral and bacterial infection was accurately detected by an eNose [46]. In another group of AECOPD patients, patients with colonisation of potentially pathogenic microorganisms had a significantly different breathprint than AECOPD patients that were not colonised. Besides, AECOPD patients' breathprints differed from stable COPD patients without microorganism colonisation [39]. Stable COPD patients with bacterial colonisation were also significantly different from those without (area under the curve (AUC) 0.922 ) [41]. Two prospective longitudinal studies indicated that the breathprint before, during and after recovery of an AECOPD differed [39, 47]. Confirming these results in larger cohort studies might lead the way to use breathprints for earlier detection and (targeted) treatment of infections and AECOPDs. This is interesting as treatment may improve outcomes and prevent hospitalizations [48].

Regarding prognostic value of eNose technology, one study demonstrated that eNose data correlated better to change in 6-min walking distance over one year, than the current GOLD classification [49]. A few studies evaluated the effect of initiation and withdrawal of inhalation medication on breathprints. Two studies found significant changes in breathprint after start of inhalation therapy $[44,50]$. A designed multidimensional model, combining eNose technology with spirometry, gave a better indication of treatment response (AUC 0.857) than spirometry only (AUC 0.561) [50]. This small pilot study shows the potential of integrating eNose technology in standard practice. However, it remains to be elucidated whether eNose technology can serve as a marker for therapy compliance of inhaled medication.

\section{Cystic fibrosis}

Cystic fibrosis (CF) is associated with bronchiectasis, recurrent infectious exacerbations, and progressive deterioration of lung function due to exacerbations [51].

A few studies using different eNoses showed that patients with CF could accurately be distinguished from healthy controls and asthma patients based on their breathprint $[23,52,53]$. Two studies showed conflicted results regarding differentiation of CF from primary ciliary dyskinesia (PCD) patients, a bronchiectatic lung disease that mimics symptoms of CF [53]. While Paff et al. showed that CF and PCD could be adequately discriminated, Joensen et al. found no significant differences $[52,53]$. This was possibly due to methodological differences, such as different breath collection methods and a more heterogeneous patient population in the latter study. Furthermore, eNose technology adequately discriminated between patients with and without exacerbations, with and without chronic Pseudomonas aeruginosa colonisation, and patients with and without Aspergillus fumigatus colonisation [52-54]. It would be of great interest to investigate whether early stage respiratory infections and exacerbations can also be detected and eventually be predicted by eNose technology. This will possibly increase the chance of successful eradication and slowing down pulmonary function decline.

\section{Interstitial lung disease}

Interstitial lung disease (ILD) is a heterogeneous group of relatively uncommon diseases causing fibrotic and/or inflammatory changes in interstitial lung tissue. Disease course and treatment strategies widely vary for different ILDs, and even within individual ILDs disease course often varies. Diagnosis is based on integration of clinical data with imaging and if needed pathology data. Diagnosis is often complex and diagnostic delays are common $[55,56]$. eNose technology has the potential to replace invasive procedures, and aid the diagnostic process to facilitate timely and accurate diagnosis.

A large single centre cohort, including various ILDs, found that breathprints of ILD patients could be distinguished from healthy controls with 100\% accuracy. Results were confirmed in a validation cohort [57]. A few other studies compared individual ILDs with healthy controls and COPD patients [58-61]. Breathprints of patients with idiopathic pulmonary fibrosis (IPF), ILD associated with connective tissue disease and pneumoconiosis were significantly different from healthy controls [59-61]. In sarcoidosis patients, the breathprint of patients with untreated sarcoidosis differed from healthy controls, implying that eNose technology may be used for initial diagnosis. This study found that breathprints of treated sarcoidosis patients were not significantly different from healthy controls, but the number of participants was small [58]. Comparing different ILDs, eNose technology distinguished IPF from non-IPF ILD patients with an accuracy of $91 \%$ in both training and validation cohort. Exploratory analyses indicated that individual ILDs can also be discriminated adequately [57]. However, groups were relatively small and, thus, results should be validated and confirmed in larger cohorts. A currently ongoing large multicentre study is investigating the potential of eNose technology to identify individual diseases, predict disease course, and response to treatment in fibrotic ILDs (NCT04680832). 


\section{Lung cancer}

Worldwide, lung cancer is the leading cause of cancer deaths and has the highest incidence of all cancer types. More than $80 \%$ of patients suffering from lung cancer are former or current tobacco smokers [62]. Early diagnosis is clearly associated with better outcomes, and lung cancer screening has shown to reduce mortality $[63,64]$. Nevertheless, early diagnosis remains challenging, since initial clinical presentation often overlaps with COPD or other smoking-related diseases, and symptoms often only appear in late stages [65]. Low-dose CT scan is currently the best available tool for screening. However, this type of screening is only cost-effective in a selected group of former and current smokers [66]. Also, differentiation of benign from malignant nodules is not possible with $\mathrm{CT}$ scan results; therefore, detected nodules warrant further invasive investigations. eNose could possibly serve as non-invasive and less costly screening tool to identify malign pulmonary neoplasms. Two studies used eNose technology in high-risk patients enrolled for lung cancer screening. Both studies found a higher specificity for detecting lung cancer with eNose compared to low-dose CT scan; thus, the use of eNose technology as screening tool can potentially reduce the false-positive rate and prevent unnecessary (invasive) testing [16, 67]. It is important to note that not all lesions classified as benign were histologically proven in these studies.

Whether an eNose can differentiate lung cancer patients from healthy controls, patients with benign lung nodules or (former) smokers, has been investigated in different cohorts. All studies in (non-) small cell lung cancer $((\mathrm{N}) \mathrm{SCLC})$ showed significant results, albeit with a wide range in reported sensitivity (71-99\%) and specificity (13-100\%) [68-80]. Smoking status of participants did not seem to influence accuracy of an eNose for detecting cancer [77]. One small study showed that patients with and without an EGFR (epidermal growth factor receptor) mutation had distinct breathprints [67]. It has not been evaluated whether eNoses can recognise specific types of lung cancer in a cohort with different subtypes. Recognition of subtypes seems plausible, as differentiation of lung cancer from head-neck cancer was possible with eNose technology [81, 82]. eNose technology did not discriminate between different stages of lung cancer [83]. One recent study in NSCLC combined eNose data with relevant clinical parameters (such as age, number of pack years, and presence of COPD), and showed a higher accuracy for lung cancer detection than using eNose data only. These results highlight the potential of eNose technology as additional diagnostic procedure [74]. Some small studies indicated that eNose technology was also able to differentiate patients suffering from malignant pleural mesothelioma (MPM) and healthy controls.
Differentiation of MPM from benign asbestosis disease and asymptomatic asbestos exposure had a high sensitivity too [84-86].

Prediction of response to therapy is investigated for anti-programmed death (PD)-1 receptor therapy in NSCLC patients. Breathprints were collected before start of pembrolizumab or nivolumab therapy. Exhaled breath data could predict which patients would respond to therapy with an AUC of 0.89 , confirmed in a validation cohort. By setting a cut-off value to obtain $100 \%$ specificity, the investigators were able to detect $24 \%$ of nonresponders to anti-PD-1 therapy. In this regard, eNose seems to be more accurate than the currently used biomarker PD-L1 [87]. Another study is currently registered for recruiting until July 2021 and will evaluate the effect of immunotherapy on breathprints of exhaled breath and sweat in lung cancer patients (NCT03988192).

Schmekel et al. investigated the ability of eNose to predict prognosis in patients with end stage lung cancer. They collected breathprints before start and several times after start of palliative chemotherapy and applied different prediction models. Patients with less than one year survival and more than one year survival could be separated based on breathprint [88]. The authors suggest to use this eNose-based prediction for choosing a certain treatment strategy, but this needs confirmation in studies first.

\section{Obstructive sleep apnoea}

At the moment, the gold standard for diagnosing obstructive sleep apnoea (OSA) is (poly)somnography which is a costly and time-consuming test. eNose technology has been investigated as an alternative modality to diagnose this condition and assess treatment effect.

It was shown that breathprints from OSA patients and healthy controls can be distinguished reliably [89-91]. However, it remains questionable whether breathprints distinguishes true OSA, or if the breathprint is just a reflection of a metabolic syndrome or underlying inflammation caused by obesity. In one of the studies this question was more apparent as groups were not matched for body mass index [89]. Dragonieri et al. found that eNose technology did discriminate obese patients with and without OSA, with moderate accuracy [90]. Nevertheless, another study could not confirm those results [91].

Other researchers investigated OSA, OSA-COPD overlap syndrome and COPD. OSA could be distinguished from the overlap syndrome, but eNose technology could not discriminate well between the overlap syndrome and COPD. Also here it is not clear whether true OSA can be detected or other factors, such as COPD, are picked up $[91,92]$. Whether included patients also suffer from heart failure is not clearly displayed in these studies, although 
it is known that many heart failure patients suffer from OSA and that heart failure might influence breathprint $[93,94]$.

The effects of continuous positive airway pressure (CPAP) treatment in patients with OSA has also been studied. The breathprint of OSA patients changed significantly already after one night of CPAP treatment [95]. Significant difference in breathprint was also found before and after three months of CPAP treatment [89]. It remains to be elucidated what this change in breathprint indicates. Possibly, the alteration in breathprint could serve as a marker for metabolic success, therapeutic benefit or treatment adherence. Furthermore, it must be noted that the breathprints of patients with OSA differed between morning and evening [96]. Hence, diurnal variance must be taken into account when using an eNose for patients with OSA.

\section{Pulmonary infections}

Pathogenic micro-organisms, such as viruses, bacteria or fungi, can cause severe pulmonary infections. Identification of specific micro-organisms with sputum cultures can take up to several days, and is only possible if a specimen with sufficient quality is obtained. Specificity and sensitivity also depend on the causative micro-organism, experience of laboratory observer, and prior treatment [97]. Therefore, reported sensitivity of detecting bacteria in sputum culture ranges between 57 and 95\%, and specificity between 48 and $87 \%$ [98]. Detection of specific micro-organisms using eNose technology can potentially reduce misuse of antibiotics and facilitate timely start of guided therapy.

Until now, two in vitro studies aimed to differentiate micro-organisms by analysing breathprints of their headspace air $[99,100]$. Mould species were discriminated from other samples (bacteria, yeasts, and control medium) with a high accuracy (92.9\%). Furthermore, different mould species seemed to have different breathprints [100]. Another study performed eNose analyses on bronchoalveolar lavage samples, and demonstrated accurate discrimination between Gram-positive bacteria, Gram-negative bacteria, fungi, and samples without growth of microorganisms [99]. In vivo, breathprints of bronchiectasis patients significantly differed between those colonised with Pseudomonas Aeruginosa and those colonised with other pathogenic micro-organisms or non-colonised [101]. For detection of aspergillus colonisation or invasive aspergillosis in specific patient groups (CF and neutropenic patients), studies revealed a high accuracy of eNose breathprint analysis [54, 102]. These studies did not include a validation cohort or healthy control group.
Ventilator-associated pneumonia (VAP) is a common nosocomial infection in ventilated patients and has an incidence and mortality around 9\% [98, 103]. In most eNose studies, bacterial growth in sputum or a clinical pneumonia score was used to define VAP [15, 104-106]. Two studies showed that obtained breathprints highly correlated with a clinical pneumonia score, implying that eNose technology might be used to predict the probability of a VAP $[104,105]$. Two case-control studies in patients with VAP and ventilated patients without pneumonia showed conflicting results; Schnabel and colleagues concluded that eNose technology lacked sensitivity and specificity, whereas a recently published study of Chen and colleagues found a good accuracy for detecting VAP $[15,106]$. This shows the need for more research on this topic before eNose can be used to determine the need for more (invasive) diagnostics in ill patients, such as performing bronchoscopy.

In pulmonary tuberculosis (TB) patients, detection and screening with eNose technology has been studied in different countries and compared to different control groups [107-112]. As TB is the leading cause of death from an infection caused by a single micro-organism, and as it has a high prevalence in developing countries, establishing a fast non-invasive cheap screening tool is much needed [113]. In one study, eNose technology differentiated TB from non-TB quite accurately, suggesting that it can potentially serve as a screening tool. Detection of TB had a sensitivity of $89 \%$ and a specificity of $91 \%$ compared to positive cultures. This sensitivity and specificity exceeded ZiehlNeelsen staining [109]. However, all studies with proven TB and healthy participants in the training cohort, had a lower accuracy when validating the results in a cohort also including suspected TB patients [107, 108, 111]. Thus, more research is necessary before eNose technology can be used as a population-wide screening tool.

Due to the Corona Virus Disease (COVID-19) pandemic, much research effort is being put in the evaluation of eNose technology as a fast and non-invasive tool for the detection of COVID-19 (NCT04475562, NCT04475575, NCT04558372, NCT04379154, NCT04614883, NL8694). To date, one study tested the accuracy of eNose technology for COVID-19 screening prior to surgery in non-symptomatic patients and found a negative predictive value up to 0.96. Reverse transcription-polymerase chain reaction on a pharyngeal swab and antibody testing were used to confirm presence or absence of COVID-19 [114].

\section{Other}

A number of eNose studies have been performed in other lung diseases. In acute respiratory distress syndrome (ARDS), eNose technology could discriminate between 
mechanically ventilated patients with and without ARDS, with moderate accuracy in a training and validation cohort [115].

One small proof-of-principle study has been performed in patients with suspected pulmonary embolism, defined as a high clinical probability according to the Well's score or elevated D-dimer. Breathprints of non-comorbid patients with and without pulmonary embolism could be distinguished with an accuracy of $85 \%$. However, in patients with comorbidities known to influence VOCs (e.g. cancer, diabetes) the accuracy dropped [116].

Finally, eNose technology could be useful for followup and monitoring lung transplant recipients. One study found a significant association between breathprint and plasma tacrolimus levels, suggesting that eNoses might be used for non-invasive therapeutic drug monitoring [117].

A clinical trial in lung transplant recipients is currently conducted (NL9251) looking at discrimination of stable lung transplant recipients, acute cellular rejection, and chronic lung allograft rejection.

\section{Discussion}

In the past decades, multiple eNoses have been developed and tested in numerous clinical studies for a wide spectrum of lung diseases. So far, the vast majority of studies evaluated the ability of eNose technology to distinguish lung diseases from healthy controls, and to discriminate between different diagnoses. A small number of studies have been performed for prognostic or therapeutic purposes, and only a handful of studies have focused on clustering patients by breathprint and identifying phenotypes. Results in lung diseases are overall very promising, but several issues should be addressed before eNoses can be implemented in daily clinical practice.

One of the issues is the use of various eNose devices with different qualifications, types of sensors and breath sample collection methods as summarised in Table 1. It is not possible to point out the best eNose device or select one optimal sensor type, as each setting, disease and research aim can require different features. For example, a portable device might be optimal for an acute care setting, direct sampling without collection bags might be useful in low resource areas and as point-of-care technique, and a device that corrects for ambient air will probably generate more comparable results in multicentre use and settings with unstable or varying environmental conditions.

Given important differences between the various devices, it is difficult to compare data of the different eNose devices. Hence, each eNose needs to be validated for every clinical application. This implies that knowledge about characteristics of eNose devices is essential before initiating eNose research, as the type of device cannot easily be changed during the trajectory of developing a clinical tool. Additionally, the influence of endogenous (e.g. comorbidities, ethnicity, age) and exogenous factors (e.g. smoking, nutrition, drug use, measurement environment) on breathprints needs to be further elucidated.

Furthermore, studies differ significantly with regards to study design (e.g. patient selection, number of participants, and presence of a validation cohort). As illustrated in Fig. 2, the majority of studies so far can be considered as pilot or exploratory studies, and have small numbers of participants. The most important goal of these studies is to test new hypotheses, which can be further assessed and confirmed in larger studies with external validation. However, these validation studies are not often conducted. This lack of validation is a major issue in development of a clinical useful breath biomarker, as breath analysis results are not always interchangeable between research settings due to a combination of the above mentioned factors. To ensure optimal outcomes, comparison and generalisability of eNose studies, the design and analysis methods should ideally be based on specific predefined research aims.

Moreover, most studies do not explain the rationale for choosing a certain machine learning model for analysing eNose data. This prevents insights in and discussion regarding the optimal analysis techniques and algorithms. Machine learning models are complex to execute and interpret, and if not used in the right way are prone for overfitting. To avoid inadequate modelling, data scientists should always be involved in these complex analyses and models should be validated independently to exclude overfitting. To allow for comparison of different modelling techniques, we recommend an extensive world-wide shared database per eNose with FAIR (findable, accessible, interoperable, and reusable) and open source data, including patient characteristics and other pre-test probabilities. This database would ensure optimal training, validation, and application of models.

Finally, a factor that hampers eNose implementation is the need for a strong gold standard to establish a diagnosis or to evaluate therapeutic effect. High quality data input is required for optimal validity when developing a new technique. Some of the diseases mentioned in this review lack a gold standard, and even if a gold standard does exist, there is always a range of uncertainty. There is a potential for unsupervised machine learning models in this regard, as such analyses could help to identify previously unrecognised phenotype clusters. Discovering such new clusters can help to generate hypotheses about the existence of unravelled disease subtypes or overlap between diagnoses, and might eventually guide new diagnostic standards. 
In conclusion, eNose technology in the field of lung diseases is promising and at the doorstep of the pulmonologist's office. To facilitate clinical implementation, we recommend conducting prospective multicentre trials including validation in external cohorts with a study design and analysis method relevant for the research aim, and sharing databases on open source platforms. If supported by sufficient evidence, research can subsequently be extended to clinical implementation studies, and finally, use in daily practice.

We believe that eNose technology has the potential to facilitate personalised medicine in lung diseases through establishing early, accurate diagnosis and monitoring disease course and therapeutic effects.

\section{Abbreviations}

AECOPD: Acute exacerbation of COPD; AUC: Area under the curve; ARDS: Acute respiratory distress syndrome; CF: Cystic fibrosis; COPD: Chronic obstructive pulmonary disease; COVID-19: Corona virus disease; EGFR: Epidermal growth factor receptor; eNose: Electronic nose; FeNO: Exhaled nitric oxide; GOLD: Global Initiative for chronic obstructive lung disease; ILD: Interstitial lung disease; IPF: Idiopathic pulmonary fibrosis; MPM: Malignant pleural mesothelioma; NSCLC: Non-small cell lung cancer; OCS: Oral corticosteroids; OSA: Obstructive sleep apnoea; PCD: Primary ciliary dyskinesia; PD: Programmed death; SCLC: Small cell lung cancer; TB: Tuberculosis; VAP:Ventilator-associated pneumonia; VOCs: Volatile organic compounds.

\section{Supplementary Information}

The online version contains supplementary material available at https://doi. org/10.1186/s12931-021-01835-4.

Additional file 1. Sensor technology explained

Additional file 2. Search strategy.

\section{Acknowledgements}

The authors wish to thank dr. Sabrina T.G. Meertens-Gunput from the Erasmus MC Medical Library for developing and updating the search strategies.

\section{Authors' contributions}

All authors contributed to conception and design of the study. NW, GN and IS conducted literature search and organised the database. NW and IS wrote the first draft of the manuscript. All authors contributed to manuscript revision, read, and approved the final manunscript.

\section{Funding}

Not applicable.

\section{Availability of data and materials}

The data supporting the conclusions of this article are included in this published article.

\section{Declarations}

Ethics approval and consent to participate

Not applicable.

\section{Consent for publication}

Not applicable.

\section{Competing interests}

Drs. Van der Sar reports grants from Boehringer Ingelheim, during the conduct of the study. Drs. Wijbenga has nothing to disclose. Drs. Nakshbandi has nothing to disclose. Dr. Aerts reports personal fees and non-financial support from MSD; personal fees from BMS, Boehringer Ingelheim, Amphera, Eli Lilly, Takeda, Bayer, Roche, Astra Zeneca outside the submitted work. In addition, Dr. Aerts has a patent on allogenic tumor cell lysate licensed to Amphera, a patent combination immunotherapy in cancer pending, and a patent biomarker for immunotherapy pending. Dr. Manintveld has nothing to disclose. Dr. Wijsenbeek reports grants and other from Boehringen Ingelheim and Hoffman la Roche, and other from Galapagos, Novartis, Respivant, Savara outside the submitted work. All grants and fees were paid to dr Wijsenbeek's institution. Dr. Hellemons has nothing to disclose. Dr. Moor reports grants and other from Boehringer-Ingelheim outside the submitted work.

\section{Author details}

'Department of Respiratory Medicine, Erasmus MC, University Medical Center, Rotterdam, The Netherlands. ${ }^{2}$ Department of Cardiology, Erasmus MC, University Medical Center, Rotterdam, The Netherlands.

Received: 1 June 2021 Accepted: 26 August 2021

Published online: 17 September 2021

\section{References}

1. van der Schee MP, Paff T, Brinkman P, van Aalderen WMC, Haarman EG, Sterk PJ. Breathomics in lung disease. Chest. 2015;147(1):224-31.

2. van de Kant KDG, van der Sande LJTM, Jöbsis Q, van Schayck OCP, Dompeling E. Clinical use of exhaled volatile organic compounds in pulmonary diseases: a systematic review. Respir Res. 2012;13(1):117.

3. Potter P. Hippocrates Vol VI. Diseases, internal affections. Cambridge, MA/London: Harvard University Press; 1988.

4. Španěl P, Smith D. Volatile compounds in health and disease. Curr Opin Clin Nutr Metab Care. 2011:14(5):455-60.

5. Lourenço C, Turner C. Breath analysis in disease diagnosis: methodological considerations and applications. Metabolites. 2014;4(2):465-98.

6. Gardner JW, Bartlett PN. A brief history of electronic noses. Sens Actuators, B Chem. 1994;18(1):210-1.

7. Liu X, Cheng S, Liu H, Hu S, Zhang D, Ning H. A survey on gas sensing technology. Sensors (Basel, Switzerland). 2012;12(7):9635-65.

8. Chen Q, Chen Z, Liu D, He Z, Wu J. Constructing E-nose using metal-ion induced assembly of graphene oxide for diagnosis of lung cancer via exhaled breath. ACS Appl Mater Interfaces. 2020. https://doi.org/10. 1021/acsami.0c00720.

9. Kovalska $\mathrm{E}$, Lesongeur $\mathrm{P}$, Hogan BT, Baldycheva A. Multi-layer graphene as a selective detector for future lung cancer biosensing platforms. Nanoscale. 2019;11(5):2476-83.

10. Nag A, Mitra A, Mukhopadhyay SC. Graphene and its sensor-based applications: a review. Sens Actuators, A. 2018;270:177-94.

11. Liu X, Cheng S, Liu H, Hu S, Zhang D, Ning H. A survey on gas sensing technology. Sensors. 2012;12(7):9635-65.

12. Santos JP, J Lozano, Aleixandre M. Electronic noses applications in beer technology. Brewing Technology, Makoto Kanauchi. IntechOpen. 2017. https://doi.org/10.5772/intechopen.68822.

13. Shobha G, Rangaswamy S. Chapter 8-Machine learning. In: Gudivada VN, Rao CR, editors. Handbook of statistics, vol. 38. Elsevier: Amsterdam; 2018. p. 197-228.

14. Liao YH, Wang ZC, Zhang FG, Abbod MF, Shih CH, Shieh JS. Machine learning methods applied to predict ventilator-associated pneumonia with Pseudomonas aeruginosa infection via sensor array of electronic nose in intensive care unit. Sensors (Basel). 2019. https://doi.org/10. 3390/s19081866.

15. Chen CY, Lin WC, Yang HY. Diagnosis of ventilator-associated pneumonia using electronic nose sensor array signals: solutions to improve the application of machine learning in respiratory research. Respir Res. 2020. https://doi.org/10.1186/s12931-020-1285-6.

16. Rocco R, Incalzi RA, Pennazza G, Santonico M, Pedone C, Bartoli IR, et al. BIONOTE e-nose technology may reduce false positives in lung cancer screening programmes. Eur J Cardio-thorac Surg. 2016;49(4):1112-7. 
17. National Asthma E, Prevention P. Expert Panel Report 3 (EPR-3): guidelines for the diagnosis and management of asthma-summary report 2007. J Allergy Clin Immunol. 2007;120(5 Suppl):S94-138.

18. Dragonieri S, Schot R, Mertens BJA, Le Cessie S, Gauw SA, Spanevello A, et al. An electronic nose in the discrimination of patients with asthma and controls. J Allergy Clin Immunol. 2007;120(4):856-62.

19. Fens $N$, Zwinderman AH, Van Der Schee MP, De Nijs SB, Dijkers E, Roldaan AC, et al. Exhaled breath profiling enables discrimination of chronic obstructive pulmonary disease and asthma. Am J Respir Crit Care Med. 2009;180(11):1076-82.

20. Lazar Z, Fens N, van der Maten J, van der Schee MP, Wagener $\mathrm{AH}$, de Nijs SB, et al. Electronic nose breathprints are independent of acute changes in airway caliber in asthma. Sensors (Basel). 2010;10(10):9127-38.

21. Montuschi P, Santonico M, Mondino C, Pennazza G, Maritini G, Martinelli $E$, et al. Diagnostic performance of an electronic nose, fractional exhaled nitric oxide, and lung function testing in asthma. Chest. 2010;137(4):790-6.

22. Van der Schee MP, Palmay R, Cowan JO, Taylor DR. Predicting steroid responsiveness in patients with asthma using exhaled breath profiling. Clin Exp Allergy. 2013;43(11):1217-25.

23. Bannier MAGE, Van De Kant KDG, Jöbsis Q, Dompeling E. Feasibility and diagnostic accuracy of an electronic nose in children with asthma and cystic fibrosis. J Breath Res. 2019. https://doi.org/10.1088/1752-7163/ aae158.

24. Dragonieri S, Quaranta VN, Carratu P, Ranieri T, Resta O. Exhaled breath profiling by electronic nose enabled discrimination of allergic rhinitis and extrinsic asthma. Biomarkers. 2019;24(1):70-5.

25. Tenero L, Sandri M, Piazza M, Paiola G, Zaffanello M, Piacentini G. Electronic nose in discrimination of children with uncontrolled asthma. J Breath Res. 2020:14(4): 046003.

26. Fens $N$, Roldaan AC, van der Schee MP, Boksem RJ, Zwinderman AH, Bel EH, et al. External validation of exhaled breath profiling using an electronic nose in the discrimination of asthma with fixed airways obstruction and chronic obstructive pulmonary disease. Clin Exp Allergy. 2011;41(10):1371-8.

27. Pembrey L, Barreto ML, Douwes J, Cooper P, Henderson J, Mpairwe $\mathrm{H}$, et al. Understanding asthma phenotypes: the World Asthma Phenotypes (WASP) international collaboration. ERJ Open Res. 2018;4(3):00013-2018

28. Farraia M, Cavaleiro Rufo J, Paciência I, Castro Mendes F, Rodolfo A, Rama T, et al. Human volatilome analysis using eNose to assess uncontrolled asthma in a clinical setting. Allergy Eur J Allergy Clin Immunol. 2020. https://doi.org/10.1111/all.14207.

29. Abdel-Aziz MI, Brinkman P, Vijverberg SJH, Neerincx AH, de Vries R, Dagelet YWF, et al. eNose breath prints as a surrogate biomarker for classifying patients with asthma by atopy. J Allergy Clin Immunol. 2020. https://doi.org/10.1016/j.jaci.2020.05.038.

30. Plaza V, Crespo A, Giner J, Merino JL, Ramos-Barbón D, Mateus EF, et al. Inflammatory asthma phenotype discrimination using an electronic nose breath analyzer. J Invest Allergol Clin Immunol. 2015;25(6):431-7.

31. Brinkman P, Wagener AH, Hekking PP, Bansal AT, Maitland-van der Zee $\mathrm{AH}$, Wang $\mathrm{Y}$, et al. Identification and prospective stability of electronic nose (eNose)-derived inflammatory phenotypes in patients with severe asthma. J Allergy Clin Immunol. 2019;143(5):1811-20.e7.

32. Brinkman P, van de Pol MA, Gerritsen MG, Bos LD, Dekker T, Smids BS, et al. Exhaled breath profiles in the monitoring of loss of control and clinical recovery in asthma. Clin Exp Allergy. 2017;47(9):1159-69.

33. Fasola S, Ferrante G, Sabatini A, Santonico M, Zompanti A, Grasso S, et al. Repeatability of exhaled breath fingerprint collected by a modern sampling system in asthmatic and healthy children. J Breath Res. 2019 https://doi.org/10.1088/1752-7163/ab1765.

34. Cavaleiro Rufo J, Paciência I, Mendes FC, Farraia M, Rodolfo A, Silva $D$, et al. Exhaled breath condensate volatilome allows sensitive diagnosis of persistent asthma. Allergy Eur J Allergy Clin Immunol. 2019:74(3):527-34.

35. Lamprecht B, Soriano JB, Studnicka M, Kaiser B, Vanfleteren LE, Gnatiuc $L$, et al. Determinants of underdiagnosis of COPD in national and international surveys. Chest. 2015;148(4):971-85.

36. Hanlon P, Daines L, Campbell C, MCKinstry B, Weller D, Pinnock H. Telehealth interventions to support self-management of long-term conditions: a systematic metareview of diabetes, heart failure, asthma, chronic obstructive pulmonary disease, and cancer. J Med Internet Res. 2017;19(5): e172.

37. Hattesohl ADM, Jörres RA, Dressel H, Schmid S, Vogelmeier C, Greulich $T$, et al. Discrimination between COPD patients with and without alpha 1-antitrypsin deficiency using an electronic nose. Respirology. 2011;16(8):1258-64.

38. Cazzola M, Segreti A, Capuano R, Bergamini A, Martinelli E, Calzetta $L$, et al. Analysis of exhaled breath fingerprints and volatile organic compounds in COPD. COPD Res Pract. 2015. https://doi.org/10.1186/ s40749-015-0010-1.

39. Shafiek H, Fiorentino F, Merino JL, López C, Oliver A, Segura J, et al. Using the electronic nose to identify airway infection during COPD exacerbations. PLoS ONE. 2015. https://doi.org/10.1371/journal.pone. 0135199.

40. Rodríguez-Aguilar M, Díaz de León-Martínez L, Gorocica-Rosete P, Padilla RP, Thirión-Romero I, Ornelas-Rebolledo O, et al. Identification of breath-prints for the COPD detection associated with smoking and household air pollution by electronic nose. Respir Med. 2020. https:// doi.org/10.1016/j.rmed.2020.105901.

41. Sibila O, Garcia-Bellmunt L, Giner J, Merino JL, Suarez-Cuartin G, Torrego A, et al. Identification of airway bacterial colonization by an electronic nose in chronic obstructive pulmonary disease. Respir Med 2014;108(11):1608-14.

42. Fens N, Van Rossum AGJ, Zanen P, Van Ginneken B, Van Klaveren RJ, Zwinderman $\mathrm{AH}$, et al. Subphenotypes of mild-to-moderate COPD by factor and cluster analysis of pulmonary function, $C T$ imaging and breathomics in a population-based survey. COPD J Chronic Obstr Pulm Dis. 2013;10(3):277-85.

43. De Vries R, Dagelet YWF, Spoor P, Snoey E, Jak PMC, Brinkman P, et al. Clinical and inflammatory phenotyping by breathomics in chronic airway diseases irrespective of the diagnostic label. Eur Respir J. 2018. https://doi.org/10.1183/13993003.01817-2017.

44. Scarlata S, Finamore P, Santangelo S, Giannunzio G, Pennazza G, Grasso $S$, et al. Cluster analysis on breath print of newly diagnosed COPD patients: effects of therapy. J Breath Res. 2018. https://doi.org/10.1088/ 1752-7163/aac273.

45. Fens N, De Nijs SB, Peters S, Dekker T, Knobel HH, Vink TJ, et al. Exhaled air molecular profiling in relation to inflammatory subtype and activity in COPD. Eur Respir J. 2011;38(6):1301-9.

46. Van Geffen WH, Bruins M, Kerstjens HAM. Diagnosing viral and bacterial respiratory infections in acute COPD exacerbations by an electronic nose: a pilot study. J Breath Res. 2016. https://doi.org/10.1088/17527155/10/3/036001.

47. van Velzen $\mathrm{P}$, Brinkman $\mathrm{P}$, Knobel HH, van den Berg JWK, Jonkers RE, Loijmans RJ, et al. Exhaled breath profiles before, during and after exacerbation of COPD: a prospective follow-up study. COPD J Chronic Obstr Pulm Dis. 2019:16(5-6): 330-337. https://doi.org/10.1080/15412 555.2019.1669550.

48. Singh D, Agusti A, Anzueto A, Barnes PJ, Bourbeau J, Celli BR, Criner GJ, Frith P, Halpin DMG, Han M, López Varela MV, Martinez F, Montes de Oca M, Papi A, Pavord ID, Roche N, Sin DD, Stockley R, Vestbo J, Wedzicha JA, Vogelmeier C. Global Strategy for the Diagnosis, Management, and Prevention of Chronic Obstructive Lung Disease: the GOLD science committee report 2019. Eur Respir J. 2019;53(5):1900164. https://doi. org/10.1183/13993003.00164-2019.

49. Finamore P, Pedone C, Scarlata S, Di Paolo A, Grasso S, Santonico M, et al. Validation of exhaled volatile organic compounds analysis using electronic nose as index of COPD severity. Int J COPD. 2018;13:1441-8.

50. Montuschi P, Santini G, Mores N, Vignoli A, Macagno F, Shoreh R, et al. Breathomics for assessing the effects of treatment and withdrawal with inhaled beclomethasone/formoterol in patients with COPD. Front Pharmacol. 2018. https://doi.org/10.3389/fphar.2018.00258.

51. Castellani C, Duff AJA, Bell SC, Heijerman HGM, Munck A, Ratjen F, et al. ECFS best practice guidelines: the 2018 revision. J Cyst Fibros. 2018;17(2):153-78.

52. Paff T, van der Schee MP, Daniels JMA, Pals G, Postmus PE, Sterk PJ, et al. Exhaled molecular profiles in the assessment of cystic fibrosis and primary ciliary dyskinesia. J Cyst Fibrosis. 2013;12(5):454-60.

53. Joensen $O$, Paff T, Haarman EG, Skovgaard IM, Jensen P $\varnothing$, Bjarnsholt $T$, et al. Exhaled breath analysis using electronic nose in cystic fibrosis and 
primary ciliary dyskinesia patients with chronic pulmonary infections. PLOS ONE. 2014. https://doi.org/10.1371/journal.pone.0115584.

54. De Heer K, Kok MGM, Fens N, Weersink EJM, Zwinderman AH, Van Der Schee MPC, et al. Detection of airway colonization by Aspergillus fumigatus by use of electronic nose technology in patients with cystic fibrosis. J Clin Microbiol. 2016;54(3):569-75.

55. Antoniou KM, Margaritopoulos GA, Tomassetti S, Bonella F, Costabel U, Poletti V. Interstitial lung disease. Eur Respir Rev. 2014;23(131):40-54.

56. Pritchard D, Adegunsoye A, Lafond E, Pugashetti JV, DiGeronimo R, Boctor $\mathrm{N}$, et al. Diagnostic test interpretation and referral delay in patients with interstitial lung disease. Respir Res. 2019;20(1):253.

57. Moor CC, Oppenheimer JC, Nakshbandi G, Aerts JGJV, Brinkman P, Maitland-van der Zee AH, et al. Exhaled breath analysis by use of eNose technology: a novel diagnostic tool for interstitial lung disease. Eur Respir J. 2021;57(1):2002042. https://doi.org/10.1183/13993003. 02042-2020.

58. Dragonieri S, Brinkman P, Mouw E, Zwinderman AH, Carratú P, Resta $\mathrm{O}$, et al. An electronic nose discriminates exhaled breath of patients with untreated pulmonary sarcoidosis from controls. Respir Med. 2013:107(7):1073-8

59. Yang HY, Peng HY, Chang CJ, Chen PC. Diagnostic accuracy of breath tests for pneumoconiosis using an electronic nose. J Breath Res. 2018. https://doi.org/10.1088/1752-7163/aa857d.

60. Krauss E, Haberer J, Maurer O, Barreto G, Drakopanagiotakis F, Degen M, et al. Exploring the ability of electronic nose technology to recognize interstitial lung diseases (ILD) by non-invasive breath screening of exhaled volatile compounds (VOC): a pilot study from the European IPF registry (eurlPFreg) and biobank. J Clin Med. 2019. https://doi.org/10. 3390/jcm8101698.

61. Dragonieri S, Scioscia G, Quaranta VN, Carratu P, Venuti MP, Falcone M, et al. Exhaled volatile organic compounds analysis by e-nose can detect idiopathic pulmonary fibrosis. J Breath Res. 2020;14(4): 047101.

62. Bray F, Ferlay J, Soerjomataram I, Siegel RL, Torre LA, Jemal A. Global cancer statistics 2018: GLOBOCAN estimates of incidence and mortality worldwide for 36 cancers in 185 countries. CA Cancer J Clin. 2018;68(6):394-424

63. Wender R, Fontham ETH, Barrera E Jr, Colditz GA, Church TR, Ettinger DS, et al. American Cancer Society lung cancer screening guidelines. CA Cancer J Clin. 2013;63(2):106-17.

64. de Koning HJ, van der Aalst CM, de Jong PA, Scholten ET, Nackaerts K, Heuvelmans MA, et al. Reduced lung-cancer mortality with volume CT screening in a randomized trial. N Engl J Med. 2020;382(6):503-13.

65. Planchard D, Popat S, Kerr K, Novello S, Smit EF, Faivre-Finn C, et al. Metastatic non-small cell lung cancer: ESMO Clinical Practice Guidelines for diagnosis, treatment and follow-up. Ann Oncol. 2018;29(Suppl 4):iv192-237.

66. Cressman S, Peacock SJ, Tammemägi MC, Evans WK, Leighl NB, Goffin $J R$, et al. The cost-effectiveness of high-risk lung cancer screening and drivers of program efficiency. J Thorac Oncol. 2017;12(8):1210-22.

67. Shlomi D, Abud M, Liran O, Bar J, Gai-Mor N, llouze M, et al. Detection of LUNG CANCER and EGFR mutation by electronic nose system. J Thorac Oncol. 2017;12(10):1544-51.

68. McWilliams A, Beigi P, Srinidhi A, Lam S, MacAulay CE. Sex and smoking status effects on the early detection of early lung cancer in high-risk smokers using an electronic nose. IEEE Trans Biomed Eng. 2015;62(8):2044-54.

69. Dragonieri S, Annema JT, Schot R, van der Schee MPC, Spanevello A, Carratú $\mathrm{P}$, et al. An electronic nose in the discrimination of patients with non-small cell lung cancer and COPD. Lung Cancer. 2009;64(2):166-70.

70. Huang $\mathrm{CH}$, Zeng C, Wang YC, Peng HY, Lin CS, Chang CJ, et al. A study of diagnostic accuracy using a chemical sensor array and a machine learning technique to detect lung cancer. Sensors (Basel). 2018. https:// doi.org/10.3390/s18092845.

71. Hubers AJ, Brinkman P, Boksem RJ, Rhodius RJ, Witte BI, Zwinderman $\mathrm{AH}$, et al. Combined sputum hypermethylation and eNose analysis for lung cancer diagnosis. J Clin Pathol. 2014:67(8):707-11.

72. Kort S, Tiggeloven MM, Brusse-Keizer M, Gerritsen JW, Schouwink $\mathrm{JH}$, Citgez E, et al. Multi-centre prospective study on diagnosing subtypes of lung cancer by exhaled-breath analysis. Lung Cancer. 2018;125:223-9.
73. van de Goor R, van Hooren M, Dingemans AM, Kremer B, Kross K. Training and validating a portable electronic nose for lung cancer screening J Thorac Oncol. 2018;13(5):676-81.

74. Kort S, Brusse-Keizer M, Gerritsen JW, Schouwink H, Citgez E, de Jongh $F$, et al. Improving lung cancer diagnosis by combining exhaled-breath data and clinical parameters. ERJ Open Res. 2020. https://doi.org/10. 1183/23120541.00221-2019.

75. Machado RF, Laskowski D, Deffenderfer O, Burch T, Zheng S, Mazzone $\mathrm{PJ}$, et al. Detection of lung cancer by sensor array analyses of exhaled breath. Am J Respir Crit Care Med. 2005;171(11):1286-91.

76. Gasparri R, Santonico M, Valentini C, Sedda G, Borri A, Petrella F, et al. Volatile signature for the early diagnosis of lung cancer. J Breath Res. 2016. https://doi.org/10.1088/1752-7155/10/1/016007.

77. Tirzite M, Bukovskis M, Strazda G, Jurka N, Taivans I. Detection of lung cancer with electronic nose and logistic regression analysis. J Breath Res. 2019. https://doi.org/10.1088/1752-7163/aae1b8.

78. Kononov A, Korotetsky B, Jahatspanian I, Gubal A, Vasiliev A, Arsenjev $A$, et al. Online breath analysis using metal oxide semiconductor sensors (electronic nose) for diagnosis of lung cancer. J Breath Res. 2020. https://doi.org/10.1088/1752-7163/ab433d.

79. Krauss E, Haberer J, Barreto G, Degen M, Seeger W, Guenther A. Recognition of breathprints of lung cancer and chronic obstructive pulmonary disease using the Aeonose ${ }^{\circledR}$ electronic nose. J Breath Res. 2020. https://doi.org/10.1088/1752-7163/ab8c50.

80. Mohamed El, Mohamed MA, Abdel-Mageed SM, Abdel-Mohdy TS, Badawi MI, Darwish SH. Volatile organic compounds of biofluids for detecting lung cancer by an electronic nose based on artificial neural network. J App Biomed. 2019;17(1):61-7.

81. van Hooren MRA, Leunis N, Brandsma DS, Dingemans AMC, Kremer B, Kross KW. Differentiating head and neck carcinoma from lung carcinoma with an electronic nose: a proof of concept study. Eur Arch Oto-Rhino-Laryngol. 2016;273(11):3897-903.

82. Fielding D, Hartel G, Pass D, Davis M, Brown M, Dent A, et al. Volatile organic compound breath testing detects in-situ squamous cell carcinoma of bronchial and laryngeal regions and shows distinct profiles of each tumour. J Breath Res. 2020;14(4): 046013.

83. Tirzite M, Bukovskis M, Strazda G, Jurka N, Taivans I. Detection of lung cancer in exhaled breath with an electronic nose using support vector machine analysis. J Breath Res. 2017. https://doi.org/10.1088/17527163/aa7799.

84. Lamote K, Brinkman P, Vandermeersch L, Vynck M, Sterk PJ, Van Langenhove $\mathrm{H}$, et al. Breath analysis by gas chromatography-mass spectrometry and electronic nose to screen for pleural mesothelioma: a crosssectional case-control study. Oncotarget. 2017;8(53):91593-602.

85. Dragonieri S, Van Der Schee MP, Massaro T, Schiavulli N, Brinkman $P$, Pinca $A$, et al. An electronic nose distinguishes exhaled breath of patients with malignant pleural mesothelioma from controls. Lung Cancer. 2012;75(3):326-31.

86. Chapman EA, Thomas PS, Stone E, Lewis C, Yates DH. A breath test for malignant mesothelioma using an electronic nose. Eur Respir J. 2012:40(2):448-54

87. De Vries R, Muller M, Van Der Noort V, Theelen WSME, Schouten RD, Hummelink K, et al. Prediction of response to anti-PD-1 therapy in patients with non-small-cell lung cancer by electronic nose analysis of exhaled breath. Ann Oncol. 2019:30(10):1660-6.

88. Schmekel B, Winquist F, Vikström A. Analysis of breath samples for lung cancer survival. Anal Chim Acta. 2014;840:82-6.

89. Greulich T, Hattesohl A, Grabisch A, Koepke J, Schmid S, Noeske S, et al. Detection of obstructive sleep apnoea by an electronic nose. Eur Respir J. 2013:42(1):145-55.

90. Dragonieri S, Porcelli F, Longobardi F, Carratu P, Aliani M, Ventura VA, et al. An electronic nose in the discrimination of obese patients with and without obstructive sleep apnoea. J Breath Res. 2015. https://doi. org/10.1088/1752-7155/9/2/026005.

91. Scarlata S, Pennazza G, Santonico M, Santangelo S, Rossi Bartoli I, Rivera $C$, et al. Screening of obstructive sleep apnea syndrome by electronicnose analysis of volatile organic compounds. Sci Rep. 2017;7(1):11938.

92. Dragonieri S, Quaranta VN, Carratu P, Ranieri T, Resta O. Exhaled breath profiling in patients with COPD and OSA overlap syndrome: a pilot study. J Breath Res. 2016. https://doi.org/10.1088/1752-7155/10/4/ 041001. 
93. Costanzo MR. Central sleep apnea in patients with heart failure-how to screen, how to treat. Curr Heart Fail Rep. 2020;17(5):277-87.

94. Finamore P, Pedone C, Lelli D, Costanzo L, Bartoli IR, De Vincentis A, et al. Analysis of volatile organic compounds: an innovative approach to heart failure characterization in older patients. J Breath Res. 2018;12(2): 026007.

95. Incalzi RA, Pennazza G, Scarlata S, Santonico M, Vernile C, Cortese L, et al. Comorbidity modulates non invasive ventilation-induced changes in breath print of obstructive sleep apnea syndrome patients. Sleep Breath. 2014. https://doi.org/10.1007/s11325-014-1065-y.

96. Kunos L, Bikov A, Lazar Z, Korosi BZ, Benedek P, Losonczy G, et al. Evening and morning exhaled volatile compound patterns are different in obstructive sleep apnoea assessed with electronic nose. Sleep Breath. 2015;19(1):247-53.

97. Skerrett SJ. Diagnostic testing for community-acquired pneumonia. Clin Chest Med. 1999;20(3):531-48.

98. Rello J, Ollendorf DA, Oster G, Vera-Llonch M, Bellm L, Redman R, et al. Epidemiology and outcomes of ventilator-associated pneumonia in a large US database. Chest. 2002;122(6):2115-21.

99. Humphreys L, Orme RML, Moore P, Charaklias N, Sahgal N, Planas Pont $\mathrm{N}$, et al. Electronic nose analysis of bronchoalveolar lavage fluid. Eur J Clin Invest. 2011;41(1):52-8.

100. De Heer K, Vonk SI, Kok M, Kolader M, Zwinderman AH, Van Oers MHJ, et al. ENose technology can detect and classify human pathogenic molds in vitro: a proof-of-concept study of Aspergillus fumigatus and Rhizopus oryzae. J Breath Res. 2016. https://doi.org/10.1088/1752-7155/ 10/3/036008

101. Suarez-Cuartin G, Giner J, Merino JL, Rodrigo-Troyano A, Feliu A, Perea $L$, et al. Identification of Pseudomonas aeruginosa and airway bacterial colonization by an electronic nose in bronchiectasis. Respir Med. 2018;136:111-7.

102. De Heer K, Van Der Schee MP, Zwinderman K, Van Den Berk IAH, Visser $C E$, Van Oers R, et al. Electronic nose technology for detection of invasive pulmonary aspergillosis in prolonged chemotherapy-induced neutropenia: a proof-of-principle study. J Clin Microbiol. 2013;51(5):1490-5.

103. Melsen WG, Rovers MM, Koeman M, Bonten MJ. Estimating the attributable mortality of ventilator-associated pneumonia from randomized prevention studies. Crit Care Med. 2011;39(12):2736-42.

104. Hanson ICW, Thaler ER. Electronic nose prediction of a clinical pneumonia score: biosensors and microbes. Anesthesiology. 2005;102(1):63-8

105. Hockstein NG, Thaler ER, Lin Y, Lee DD, Hanson CW. Correlation of pneumonia score with electronic nose signature: a prospective study. Ann Otol Rhinol Laryngol. 2005;114(7):504-8.

106. Schnabel RM, Boumans MLL, Smolinska A, Stobberingh EE, Kaufmann R, Roekaerts PMHJ, et al. Electronic nose analysis of exhaled breath to diagnose ventilator-associated pneumonia. Respir Med. 2015;109(11):1454-9.
107. Bruins M, Rahim Z, Bos A, van de Sande WW, Endtz HP, van Belkum A. Diagnosis of active tuberculosis by e-nose analysis of exhaled air. Tuberculosis (Edinb). 2013;93(2):232-8.

108. Coronel Teixeira R, Rodríguez M, Jiménez de Romero N, Bruins M, Gómez R, Yntema JB, et al. The potential of a portable, point-of-care electronic nose to diagnose tuberculosis. J Infect. 2017;75(5):441-7.

109. Fend R, Kolk AHJ, Bessant C, Buijtels P, Klatser PR, Woodman AC. Prospects for clinical application of electronic-nose technology to early detection of Mycobacterium tuberculosis in culture and sputum. J Clin Microbiol. 2006;44(6):2039-45.

110. Mohamed El, Mohamed MA, Moustafa MH, Abdel-Mageed SM, Moro AM, Baess Al, et al. Qualitative analysis of biological tuberculosis samples by an electronic nose-based artificial neural network. Int J Tuberc Lung Dis. 2017;21(7):810-7.

111. Saktiawati AMI, Stienstra Y, Subronto YW, Rintiswati N, Sumardi JWG, et al. Sensitivity and specificity of an electronic nose in diagnosing pulmonary tuberculosis among patients with suspected tuberculosis. PLoS ONE. 2019. https://doi.org/10.1371/journal.pone.0217963.

112. Zetola NM, Modongo C, Matsiri O, Tamuhla T, Mbongwe B, Matlhagela $\mathrm{K}$, et al. Diagnosis of pulmonary tuberculosis and assessment of treatment response through analyses of volatile compound patterns in exhaled breath samples. J Infect. 2017;74(4):367-76.

113. World Health Organization. https://www.who.int/news-room/factsheets/detail/tuberculosis.

114. Wintjens AGWE, Hintzen KFH, Engelen SME, Lubbers T, Savelkoul PHM, Wesseling G, van der Palen JAM, Bouvy ND. Applying the electronic nose for pre-operative SARS-CoV-2 screening. Surg Endosc. 2020:1-8. https://doi.org/10.1007/s00464-020-08169-0.

115. Bos LDJ, Schultz MJ, Sterk PJ. Exhaled breath profiling for diagnosing acute respiratory distress syndrome. BMC Pulm Med. 2014. https://doi. org/10.1186/1471-2466-14-72.

116. Fens N, Douma RA, Sterk PJ, Kamphuisen PW. Breathomics as a diagnostic tool for pulmonary embolism. J Thromb Haemost. 2010;8(12):2831-3.

117. Kovacs D, Bikov A, Losonczy G, Murakozy G, Horvath I. Follow up of lung transplant recipients using an electronic nose. J Breath Res. 2013. https://doi.org/10.1088/1752-7155/7/1/017117.

118. Abdel-Aziz M, Brinkman P, Vijverberg S, Neerincx A, Vries R, Dagelet $Y$, et al. eNose breathprints as a surrogate biomarker for classifying asthma patients by atopy. J Allergy Clin Immunol. 2020.

\section{Publisher's Note}

Springer Nature remains neutral with regard to jurisdictional claims in published maps and institutional affiliations. 